
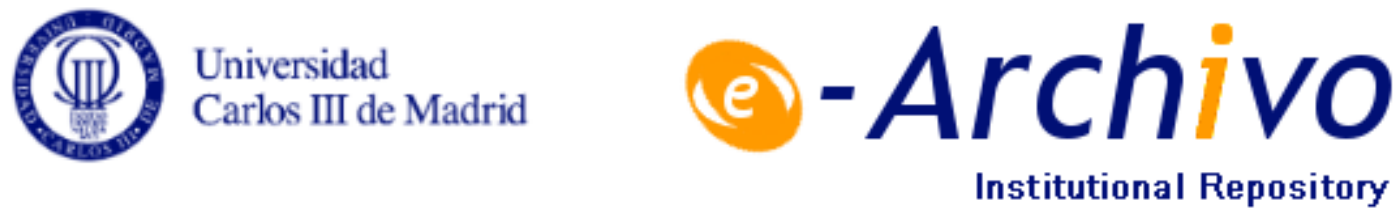

This is a postprint version of the following published document:

Josa-Fonbellida, R., Rincón-Zapatero, J.P (2015). Euler-Lagrange equations of stochastic differential games: application to a game of a productive asset. Economic Theory, v. 59, n. 1, pp. 61-108. Available in: https://doi.org/10.1007/s00199-015-0873-z

(C) Springer Verlag 


\title{
Euler-Lagrange equations of stochastic differential games: application to a game of a productive asset
}

\author{
Ricardo Josa-Fombellida ${ }^{1}$ • Juan Pablo Rincón-Zapatero
}

\begin{abstract}
This paper analyzes a noncooperative and symmetric dynamic game where players have free access to a productive asset whose evolution is a diffusion process with Brownian uncertainty. A Euler-Lagrange equation is found and used to provide necessary and sufficient conditions for the existence and uniqueness of a smooth Markov Perfect Nash Equilibrium. The Euler-Lagrange equation also provides a stochastic Keynes-Ramsey rule, which has the form of a forward-backward stochastic differential equation. It is used to study the properties of the equilibrium and to make some comparative statics exercises.
\end{abstract}

Keywords Stochastic productive asset - Markov Perfect Nash Equilibrium . Euler-Lagrange equations

JEL Classification $\quad$ C73 $\cdot$ C61

\begin{abstract}
We gratefully acknowledge the constructive comments of the anonymous referees and the editor. Part of this work was done while Juan Pablo Rincón-Zapatero was visiting the Department of Economics at Indiana University with the support of a Salvador de Madariaga Scholarship. The authors were also supported by the Spanish Ministerio de Ciencia e Innovación under Projects ECO2008-02358 and ECO2011-24200, and first author from Junta de Castilla y León under Project VA056A09.
\end{abstract}

$凶$ Juan Pablo Rincón-Zapatero

jrincon@eco.uc3m.es

Ricardo Josa-Fombellida

ricar@eio.uva.es

1 Departamento de Estadística e Investigación Operativa and IMUVA, Universidad de Valladolid, P. de Belén 7, 47011 Valladolid, Spain

2 Departamento de Economía, Universidad Carlos III de Madrid, C/ Madrid, 126, 28903 Getafe, Madrid, Spain 


\section{Introduction}

The purpose of this paper is twofold. On the one hand, we present a method to study stochastic differential games which is reminiscent of the Euler-Lagrange equations (EL henceforth) of the Calculus of Variations problem. On the other hand, we study with this approach a differential game where $N$ identical agents compete on consumption of a productive asset, proving the existence and the uniqueness of symmetric equilibrium. We also analyze some of its main properties. The model we study is also important in the single-player case, as the game reduces to the one-sector stochastic growth model analyzed in Bourguignon (1974) and Merton (1975). Despite its simplified nature, this model is fundamental to understand the dynamics of consumption and capital formation in a one-sector economy where labor supply is affected by an additive noise. Moreover, in the two-person case, the model is a stochastic version of the differential game of capitalism introduced by Lancaster (1973), but where the production factors are fully employed, as in Shimomura (1991).

EL equations are one of the more useful tools to study dynamic optimization problems. Introduced in the Calculus of Variations for the first time, EL equations have become a cornerstone in dynamic economic analysis. They provide a first-order optimality condition for interior solutions of dynamic problems, avoiding the use of the value function. The value function is characterized by the Hamilton-Jacobi-Bellman equation (HJB henceforth). HJB equations provide a general characterization of optimality, in the sense that they do not need interiority of the equilibrium to hold. ${ }^{1}$ Once the value functions are known, the Markov Perfect Nash Equilibrium (MPNE henceforth) is recovered from the HJB equation as the fixed point of the best response mappings in the Hamiltonian game. ${ }^{2}$ It is worth noting that the resolution of the EL equations provides the solution directly, with no need to compute the value function. In fact, the value function can be found once the EL equations are solved, as we will show below.

What we propose in this paper is a general method for obtaining EL equations in a class of stochastic differential games, in which the MPNE is interior, and the uncertainty, which is modeled as a standard Brownian motion, is independent of the strategies of the players. This is the most serious limitation of the method developed in the paper. Nevertheless, many interesting economic models other than those from finance present this feature as games of Cournot competition with additive noise in demand, games of dynamic provision of public goods, advertising games, or the noncooperative game of exploitation of a productive asset that we study here.

The idea of obtaining the EL equations in a differential game to determine the MPNE can be traced back to Case (1974), where this author studies a deterministic differential game of Cournot competition. To find the solution, a system of differential equations that characterizes the Nash equilibrium is found. This corresponds to what we call in our paper EL equations. Tsutsui and Mino (1990) also uses the EL equation to find infinitely many discontinuous MPNE in an oligopolistic differ-

\footnotetext{
${ }^{1}$ EL equations can also be formulated in the general case by introducing multipliers and inequalities instead of equalities, although obviously in this case, they lose much of their direct applicability.

2 Of course, this program succeeds in continuous time under some technical conditions guaranteeing the existence of solutions of the corresponding evolution equations.
} 
ential game. Further concrete applications of the use of the EL equations system in differential game theory can be found in Shimomura (1991), Dockner and Sorger (1996), and Sorger (1998), all of them in the deterministic counterpart of the model we study in this paper. In Rincón-Zapatero et al. (1998) and Rincón-Zapatero (2004), this approach has been made systematic. These two papers also provide sufficient conditions of optimality which are independent of the value function. An application to a general model of exploitation of a nonrenewable resource in a finite horizon is given. In Martín-Herrán and Rincón-Zapatero (2005), the EL equations are used to identify games of fishery where the MPNE is Pareto optimal. The subsequent paper Josa-Fombellida and Rincón-Zapatero (2007) focuses on a stochastic control problem with Brownian uncertainty, where the players' decisions cannot affect the size of the uncertainty. ${ }^{3}$ The present paper extends this methodology to the game framework. It is worth commenting on the recent paper Dockner and Wagener (2014), where a similar approach is used for deterministic games, but based on a differential equation satisfied by the costate variable in feedback form.

It is well known that the question of existence of equilibria in stochastic differential games is not satisfactorily addressed by the theory, and, in particular, the development of this issue is well behind the corresponding theory in continuous stochastic games with simultaneous moves and discrete time. Amir (1996a) shows, using Topkin's Theorem and supermodularity, the existence and uniqueness of equilibria in non-symmetric stochastic games of capital accumulation with a convex transition technology. The result is applied to a model of altruistic growth in Amir (1996b). In this framework, Nowak (2006) dispenses the supermodularity condition on the utility function and Balbus et al. (2014) extends these results to allow for more general spaces of strategies, providing constructive methods to find the equilibrium. All these papers use a powerful idea in Amir (1996a, 1997), considering convex combinations of transition probabilities. This method of averaging regularizes the best response mapping (or correspondence), enabling the introduction of more regular strategy spaces than those needed in the deterministic counterpart, see Sundaram (1989). In this way, the SchauderTikhonov fixed point theorem can be invoked to prove existence of equilibrium in the class of Lipschitz functions. A similar phenomenon happens in continuous time. The consideration of a continuous Brownian motion as the source of uncertainty has the effect of regularizing the problem, so that an smooth equilibrium arises (in the symmetric case). This effect is well known in the theory of partial differential equations (PDEs henceforth): addition of a term involving second derivatives to a first-order equation (this corresponds to a deterministic problem) leads to higher regularity of the solutions.

Our results of existence and uniqueness are based on classical theorems on PDEs that cannot be applied directly, but have to be adapted to cover the specificities of the economic model. What we do is to apply those theorems on bounded subdomains, providing uniform lower and upper bounds on the solutions. After this, the global solution

\footnotetext{
3 EL equations were found following the stochastic maximum principle conditions, which is a more general way than that used here, based on the value function; the second approach has the advantage of simplicity. In Josa-Fombellida and Rincón-Zapatero (2010), an EL equation of a Mayer problem, where the diffusion coefficient depends on the control, has been obtained and analyzed. It turns out that the EL equation in this case is much more complex.
} 
is obtained after passing to the limit. What is new in our use of the classical theorems is the following: (1) the transformation of the equation by means of an auxiliary function that gives the aforementioned bounds and (2) to get these bounds by using the maximum principle for parabolic PDEs, to obtain these bounds for minimal and maximal solutions by using the maximum principle for parabolic PDEs and Daskin's Theorem. To get sharp bounds of the solution is essential to prove that the solution of the PDE is also a MPNE, as well as to show the convergence of the finite-horizon MPNE to the infinite-horizon one. Contrary to the infinite-horizon case, the finite-horizon PDE is a well-defined Cauchy problem, thus the solution could be computed with standard PDE algorithms, providing a method to get approximations to the infinite-horizon equilibrium.

The exploitation of resources under noncooperative management has received much attention in the literature. The recent survey of Long (2011) provides a modern and exhaustive account. Origins of the literature can be attached to the seminal contribution of Levhari and Mirman (1980), where a discrete time version of the game is studied. They find an explicit solution of the equilibrium when the players have logarithm utilities. The equilibrium leads to an inefficient allocation of resources since, as a consequence of the noncooperative character of the game, the resource is overexploited. Further studies in the field are Clemhout and Wan (1985), Sundaram (1989), Benhabib and Radner (1992), Dutta and Sundaram (1993a,b), Kamihigashi (2006), Mitra and Roy (2006), Dockner and Sorger (1996), and Sorger (1998), among others. Of special relevance to us are the two latter papers. Both show that with an infinite horizon, infinitely many subgame perfect equilibria of the symmetric game exist. All of them are discontinuous with respect to the asset stock level. The reason is the assumption taken with respect to the elasticity of the marginal utility, which says that it is greater than-Sorger (1998) — or equal—Dockner and Sorger (1996) — to the ratio $N /(N-1)$, where $N$ is the number of players. ${ }^{4}$ The willingness of the players to exchange consumption between time periods strengthens the fight for the resource and, as we prove, it makes the consumption rate jump to infinite, no matter the resource suffers stochastic fluctuations. To avoid this problem, the aforementioned papers impose an exogenous upper bound on the consumption rate. We consider here just the opposite assumption, that is, that the willingness to exchange consumption across time is strictly less than $N /(N-1)$.

As in most of the previous literature, we focus on the symmetric game and the symmetric Nash equilibrium. Another feature of our study is that we analyze mainly games with a finite horizon, allowing for rather general bequest functions at the final time. Then, we show how the finite-horizon equilibrium approaches the infinite-horizon one as the horizon tends to infinite for an ample class of bequest functions. Finite-horizon games serve to model problems where access to the fishing pool is valid only for a fixed period of time; after the time expires, the bequest function of each player could represent some tax that the owner of the resource imposes on the players for the use of the resource over the agreed period of time.

We prove the existence and uniqueness of smooth MPNE for the finite-horizon game, under quite general hypotheses, allowing for the unboundedness of the func-

\footnotetext{
${ }^{4}$ Note that in particular the one-player game never fulfills these assumptions.
} 
tions intervening in the definition of the problem, as well as non-Lipschitzianity of the utility function. This is necessary to cover the more popular cases, including those that allow an analytical resolution. However, no specific functional form is postulated in our results.

A consequence of the EL equation is a stochastic Keynes-Ramsey rule (KR rule henceforth) that governs the equilibrium. Due to the stochastic nature of the problem, the rule consists of a pair of forward-backward stochastic differential equations. In the single-player deterministic case, the rule was first established for the optimal growth model analyzed in Ramsey (1928). It characterizes the rate of change of players' optimal consumption in response to changes in the interest rate and in the elasticity of intertemporal substitution. In the stochastic case, the expected rate of change in consumption is much more complex. New terms appear that contain the stochastic characteristics of the model, as well as the prudence attitude of the players. We find that a higher prudence index leads to a higher mean increment in consumption. We also prove a general result on the overall effect of uncertainty and show that under the standard hypothesis of concavity on the bequest and the recruitment functions, the larger the uncertainty, the larger the consumption rate of the players. Thus, uncertainty sharpens competition among players. ${ }^{5}$ It turns out that the KR rule is very useful in doing some exercises on comparative statics, studying the effect on the equilibrium of a variation in the number of players and in the time preference rate.

We also study the curvature of the equilibrium, finding conditions such that the consumption equilibrium is concave with respect to the state variable. The curvature of the consumption rule gives us information concerning players' propensity to consume. A concave consumption rule implies a higher propensity to consume for poor people than for rich people. Carroll and Kimball (1996) proved concavity of the consumption function in a one-player game of finite horizon and discrete time, where uncertainty comes from three sources: labor income, gross interest rate, and discount factor. The family of utility functions considered by those authors were of the constant relative risk aversion class (CRRA henceforth), strictly increasing, concave, and with convex marginal utility. We consider only one source of uncertainty, but use continuous time and allow for a rather general bequest function, whereas Carroll and Kimball (1996) consider no bequest function at the end of the game. We obtain definite results only for the game we call the linear game, which will be defined below.

Finally, we analyze extinction, that is, whether the competition could lead to an overexploitation of the resource and eventually drive the resource stock to zero. It is also worth comparing the stochastic case we deal with here and the deterministic case, analyzed in Clemhout and Wan (1985). We show that uncertainty raises the possibility of extinction.

\footnotetext{
5 Note that this claim has no direct implications for the precautionary savings behavior of players, as the context is different. Precautionary savings means that the agent saves more today when there is uncertainty in his/her tomorrow's income than when the uncertainty is eliminated by adding the expected income to the wealth process. We do not carry out this exercise here, but compare the Markov equilibrium strategies of two games with different diffusion coefficients, without modifying the income process (in this case, the productive asset process). Usually, precautionary savings appear when the marginal utility is convex, which can be easily proved in two period, discrete time models by means of Jensen's inequality, see Leland (1968).
} 
The paper has the following structure. Section 2 contains the definition of a general stochastic game where player's actions do no affect the size of uncertainty, as well as the definition of admissible strategies and of the MPNE. Then comes the deduction of the EL equation system from the HJB equation. Section 3 presents the game of exploitation of an stochastic productive asset and the associated EL equations. This section also contains the basic assumptions we need to attain our results and a characterization of a family of utility functions that fulfill them. In Sect. 4, we prove the existence and uniqueness of MPNE in two steps: first, we prove existence and uniqueness-within a given class of functions - of solution to the EL equation by extending classical results from the PDE theory to our framework. These technical results are relegated to "Appendix"; second, we prove that the solution of the PDE is in fact an MPNE of the game. Some insights into the infinite-horizon game are also provided. Section 5 focuses on the KR rule, and Sect. 6 is devoted to study some properties of the equilibrium. Besides the comparative statics of the equilibrium, we obtain a turnpike result and analyze the question of the extinction of the resource. Finally, some conclusions are extracted from the paper in Sect. 7. "Appendix" contains the results about PDEs commented above, as well as other proofs that we consider of a technical character.

\section{Description of the game and Euler-Lagrange equations}

In this section, we formulate a general stochastic differential game to which the model studied in the paper is a particular case. Standard references for differential games are Melhmann (1988), Başar and Olsder (1999), or Dockner et al. (2000).

We shall use the following notation. The partial derivatives are indicated by subscripts and $\partial_{x}$ stands for total derivation; the partial derivative of a scalar function with respect to a vector is a column vector; given a real vector function $g: \mathbb{R}^{n} \longrightarrow \mathbb{R}^{m}$ and a vector $z \in \mathbb{R}^{n}, g_{z}$ is defined as the matrix $\left(\partial g^{i} / \partial z^{j}\right)_{i, j}$; for a matrix $A, A^{(i)}$ denotes the $i$ th column and $A^{i j}$ denotes the $(i, j)$ element; vectors $v \in \mathbb{R}^{n}$ are column vectors and $v^{i}$ is the $i$ th component; ${ }^{\top}$ denotes transposition.

We consider an $N$-person differential game over a fixed and bounded time interval $[0, T]$ with $0<T \leq \infty$. Let $(\Omega, \mathcal{F}, \mathbf{P})$ be a complete probability space. Assume that on this space, a $d$-dimensional Brownian motion $\left\{w(t), \mathcal{F}_{t}\right\}_{t \in[0, T]}$ is defined with $\left\{\mathcal{F}_{t}\right\}_{t \in[0, T]}$ being the Brownian filtration. Let $\mathrm{E}$ denote expectation under the probability measure $\mathbf{P}$. We also consider the function space $L_{\mathcal{F}}^{2}\left([0, T] ; \mathbb{R}^{n}\right)$ of all processes $X(\cdot)$ with values in $\mathbb{R}^{n}$ adapted to filtration $\left\{\mathcal{F}_{t}\right\}_{t \geq 0}$ such that $\mathrm{E} \int_{0}^{T}\|X(t)\|^{2} d t<\infty$.

The state space is a subset $\mathcal{X} \subseteq \mathbb{R}^{n}$ and the set of admissible profiles of the players is some subset $U=U^{1} \times U^{2} \times \cdots \times U^{N}$, where $U^{i} \subseteq \mathbb{R}^{m_{i}}$, with ${ }^{6} m_{i}=$ $n$, for all $i=1, \ldots, N$. A $U$-valued process of strategic profiles $\left\{\left(u(s) ; \mathcal{F}_{s}\right)=\right.$ $\left.\left(\left(u^{1}(s), u^{2}(s), \ldots, u^{N}(s)\right) ; \mathcal{F}_{s}\right)\right\}$ defined on $[t, T] \times \Omega$ is an $\mathcal{F}_{s}$-progressively measurable map $(r, \omega) \rightarrow u(r, \omega)$ from $[t, s] \times \Omega$ into $U$, that is, $u(t, \omega)$ is $\mathcal{B}_{s} \times \mathcal{F}_{s}-$ measurable for each $s \in[t, T]$, where $\mathcal{B}_{s}$ denotes the Borel $\sigma$-field in $[t, s]$. For simplicity, we will denote $u(t, \omega)$ by $u(t)$.

\footnotetext{
6 The case $m_{i}>n$ could also be analyzed, by means of a reduction to the case $m_{i}=n$ as in Josa-Fombellida and Rincón-Zapatero (2007).
} 
The state process $X \in \mathbb{R}^{n}$ satisfies the system of controlled stochastic differential equations (SDEs henceforth)

$$
d X(s)=f(s, X(s), u(s)) d s+\sigma(s, X(s)) d w(s), \quad t \leq s \leq T,
$$

with initial condition $X(t)=x, t \in[0, T], x \in \mathbb{R}^{n}$. Observe that the diffusion coefficient, $\sigma$, is independent of the control variable, $u$. The functions $f:[0, T] \times$ $\mathbb{R}^{n} \times U \longrightarrow \mathbb{R}^{n}$ and $\sigma:[0, T] \times \mathbb{R}^{n} \longrightarrow \mathbb{R}^{n \times d}$ are both assumed to be of class $\mathcal{C}^{2}$ with respect to $(x, u)$ and of class $\mathcal{C}^{1}$ with respect to $t$. Since our aim is to work with the MPNE concept, we will consider the game for every initial condition $(t, x)$.

Definition 1 (Admissible strategies) A strategic profile $\left\{\left(u(t) ; \mathcal{F}_{t}\right)\right\}_{t \in[0, T]}=\left\{\left(\left(u^{1}(t), u^{2}(t), \ldots, u^{N}(t)\right) ; \mathcal{F}_{t}\right)\right\}_{t \in[0, T]}$ is called admissible if

(i) for every $(t, x)$ the system of SDEs (1) with initial condition $X(t)=x$ admits a pathwise unique strong solution;

(ii) for each $i=1, \ldots, N$, there exists some function $\phi^{i}:[0, T] \times \mathbb{R}^{n} \longrightarrow U^{i}$ of class $\mathcal{C}^{1,2}$ with respect to $(t, x)$ such that $u^{i}$ is in relative feedback to $\phi^{i}$, i.e. $u^{i}(s)=\phi^{i}(s, X(s))$ for every $s \in[0, T]$.

Let $\mathcal{U}^{i}(t, x)$ denote the set of admissible strategies of player $i$ and $\mathcal{U}=\mathcal{U}^{1} \times \cdots \times \mathcal{U}^{N}$ the set of admissible strategies profiles, corresponding to the initial condition $(t, x) \in$ $[0, T] \times \mathbb{R}^{n}$.

Part (ii) in Definition 1 means that players use Markov strategies. When $\phi^{i}$ is time independent, we will say that the strategy is a stationary Markovian strategy.

The instantaneous utility function of player $i$ is denoted by $L^{i}$ and his or her bequest function by $S^{i}$. Given initial conditions $(t, x) \in[0, T] \times \mathbb{R}^{n}$ and an admissible strategic profile $u$, the payoff function of each player (to be maximized) is given by

$$
J^{i}(t, x ; u)=\mathrm{E}_{t x}\left\{\int_{t}^{T} e^{-r_{i}(s-t)} L^{i}(s, X(s), u(s)) d s+e^{-r_{i}(T-t)} S^{i}(T, X(T))\right\},
$$

where $\mathrm{E}_{t x}$ denotes conditional expectation with respect to the initial condition $(t, x)$. In the following, the subscript will be eliminated if there is no confusion. The functions $L^{i}:[0, T] \times \mathbb{R}^{n} \times U \longrightarrow \mathbb{R}$ and $S^{i}:[0, T] \times \mathbb{R}^{n} \longrightarrow \mathbb{R}, i=1, \ldots, N$, are both of class $\mathcal{C}^{2}$ with respect to $(x, u)$ and of class $\mathcal{C}^{1}$ with respect to $t$. The constant $r_{i} \geq 0$ is the rate of discount. $J^{i}(t, x ; u)$ denotes the utility obtained by player $i$ when the game starts at $(t, x)$ and the profile of strategies is $u$. Given that our aim is to solve the problem for every $(t, x) \in[0, T] \times \mathbb{R}^{n}, \mathcal{U}$ will often be written instead of $\mathcal{U}(t, x)$.

In the infinite-horizon case, the bequest functions $S^{i}$ are null. In this case, if the problem is autonomous and the strategies are Markov stationary, the value function is independent of time, and the initial condition is simply $x$.

In a noncooperative setting, the aim of the players is to maximize their individual payoff $J^{i}$. Since this aspiration depends on the strategies selected by the other players also, it is generally impossible to attain ${ }^{7}$. An adequate concept of solution is Nash equi-

\footnotetext{
7 But in some models, the MPNE is also Pareto optimal; see Martín-Herrán and Rincón-Zapatero (2005).
} 
librium, which prevents unilateral deviations of the players from its recommendation of play.

Definition $2(M P N E)$ An $N$-tuple of strategies $\widehat{\phi} \in \mathcal{U}$ is called a Markov Perfect Nash Equilibrium if for every $(t, x) \in[0, T] \times \mathbb{R}^{n}$, for every $\phi^{i} \in \mathcal{U}^{i}$

$$
J^{i}\left(t, x ;\left(\phi^{i} \mid \widehat{\phi}_{-i}\right)\right) \leq J^{i}(t, x ; \widehat{\phi}),
$$

for all $i=1, \ldots, N$.

In the above definition, $\left(\phi^{i} \mid \widehat{\phi}_{-i}\right)$ denotes $\left(\widehat{\phi}^{1}, \ldots, \widehat{\phi}^{i-1}, \phi^{i}, \widehat{\phi}^{i-1}, \ldots, \widehat{\phi}^{N}\right)$. Note that with an MPNE, no player has incentives to deviate unilaterally from the equilibrium, whatever the initial condition $(t, x)$ is.

Definition 3 (Value functions) Let $\widehat{\phi}$ be an MPNE of the game. The value function $V^{i}$ of the $i$ th player is

$$
\begin{gathered}
V^{i}(t, x)=\sup _{\phi^{i} \in \mathcal{U}^{i}}\left\{J^{i}\left(t, x ;\left(\phi^{i} \mid \widehat{\phi}_{-i}\right)\right): d X(s)=f\left(s, X(s),\left(\phi^{i} \mid \widehat{\phi}_{-i}\right)(s, X(s))\right) d s\right. \\
+\sigma(s, X(s)) d w(s), X(t)=x, \quad \forall s \in[t, T]\},
\end{gathered}
$$

for all $(t, x) \in[0, T] \times \mathbb{R}^{n}$, for all $i=1, \ldots, N$.

Theorem 1 (Euler-Lagrange equations) Suppose that for all $i=1, \ldots, N, V^{i}$ and $\phi^{i}$ are of class $C^{1,2}$, that $V_{x t}^{i}=V_{t x}^{i}$ and that $\phi^{i}$ is interior to the control region $U^{i}$. Then $\left(\phi^{1}, \ldots, \phi^{N}\right)$ satisfy the system of PDEs

$$
\begin{aligned}
r_{i} \Lambda^{i}(t, x, \phi)= & \frac{\partial}{\partial t} \Lambda^{i}(t, x, \phi)+\frac{\partial}{\partial x} H^{i}\left(t, x, \phi, \Lambda^{i}(t, x, \phi)\right) \\
& +\frac{1}{2} \frac{\partial}{\partial x} \operatorname{Tr}\left(\sigma \sigma^{\top} \frac{\partial}{\partial x} \Lambda^{i}(t, x, \phi)\right),
\end{aligned}
$$

with final conditions $\phi^{i}(T, x)=\varphi^{i}(x)$ given implicitly by

$$
L_{u^{i}}^{i}\left(T, x, \varphi^{1}(x), \ldots, \varphi^{N}(x)\right)+S_{x}^{i}(T, x) f_{u^{i}}\left(T, x, \varphi^{1}(x), \ldots, \varphi^{N}(x)\right)=0,
$$

for $i=1, \ldots, N$.

Proof The smoothness of the value functions of the players guarantee that they satisfy the HJB equations with terminal conditions

$$
\begin{aligned}
r_{i} V^{i}(s, x) & =V_{t}^{i}(s, x)+\max _{u^{i} \in U^{i}} H^{i}\left(s, x,\left(u^{i} \mid \phi_{-i}\right), V_{x}^{i}(s, x)\right)+\frac{1}{2} \operatorname{Tr}\left(\sigma \sigma^{\top} V_{x x}^{i}\right)(s, x), \\
V^{i}(T, x) & =S^{i}(T, x), \quad t \leq s \leq T,
\end{aligned}
$$

where $H^{i}$ is the Hamiltonian of player $i$

$$
H^{i}\left(s, x, u, \lambda^{i}\right)=L^{i}(s, x, u)+\left(\lambda^{i}\right)^{\top} f(s, x, u) .
$$


Since the MPNE is interior to $U=U^{1} \times \cdots \times U^{N}$, the maximization condition

$$
\max _{u^{i} \in U^{i}} H^{i}\left(t, x,\left(u^{i} \mid \phi_{-i}\right), V_{x}^{i}\right), \quad i=1, \ldots, N
$$

turns into

$$
L_{u^{i}}^{i}\left(s, x,\left(\phi^{i} \mid \phi_{-i}\right)\right)+f_{u^{i}}\left(s, x,\left(\phi^{i} \mid \phi_{-i}\right)\right)^{\top} V_{x}^{i}=0, \quad i=1, \ldots, N
$$

which is explicitly solvable for $V_{x}^{i}=\Lambda^{i}(s, x, \phi):=f_{u^{i}}^{-\top} L_{u^{i}}^{i}(t, x, \phi)$, supposing $f_{u}^{i}$ to be invertible for all $i$. The above equality also holds at time $t=T$. Since $V(T, x)=S(x), V_{x}(T, x)=S^{\prime}(x)$. Plugging this value into the equality, we get the final condition (3). On the other hand, by the envelope theorem, for each $j=1, \ldots, n$ we obtain

$$
r_{i} V_{x_{j}}^{i}(t, x)=V_{x_{j} t}^{i}(t, x)+\frac{\partial}{\partial x_{j}} H^{i}\left(t, x, \phi, V_{x}^{i}(t, x)\right)+\frac{1}{2} \frac{\partial}{\partial x_{j}} \operatorname{Tr}\left(\sigma \sigma^{\top} V_{x x}^{i}(t, x)\right) .
$$

Substituting now $V_{x}^{i}=\Lambda^{i}(t, x, \phi)$ we get for the MPNE $\phi=\left(\phi^{1}, \ldots, \phi^{N}\right)$ the EL system of equations of differential type given in (2).

Notice that $\partial / \partial x$ denotes total differential with respect to $x$. No explicit dependence of the value functions appears, as in the EL equations in discrete dynamic programming.

\section{Competition for consumption of a stochastic productive asset: game, assumptions, and Euler-Lagrange equations}

\subsection{The game}

We consider a continuous time noncooperative game where $N$ agents consume a stochastic productive asset. Asset stock at time $t \geq 0$ is denoted by $X(t)$ and the consumption rate of player $i \in\{1, \ldots, N\}$, denoted $c^{i}(t)$, is given through a Markov strategy $\phi^{i}:[0, T] \times[0, \infty) \longrightarrow[0, \infty)$, that is, $c^{i}(t)=\phi^{i}(t, X(t)) .^{8}$ Given a consumption profile of Markov strategies of the other players, $\phi_{-i}=$ $\left(\phi^{1}, \ldots, \phi^{i-1}, \phi^{i+1}, \ldots, \phi^{N}\right)$, player $i$ chooses consumption $c^{i} \geq 0$ to maximize his/her payoff

$$
J_{i}\left(t, x, \phi_{-i} ; c^{i}\right)=\mathrm{E}_{t x} \int_{t}^{T} e^{-r_{i}(s-t)} L_{i}\left(c^{i}(s)\right) d s+e^{-r_{i}(T-t)} \mathrm{E}_{t x} S_{i}(X(T)) .
$$

This is given by the expected total utility of consumption, $L_{i}\left(c^{i}\right)$, over a fixed time horizon $[0, T]$, plus the utility derived from the asset stock at the end of the period,

\footnotetext{
8 The game is a particular case of the general framework described in Sect. 2 above. The class of admissible strategies is given in Definition 1.
} 
$S_{i}$, both discounted at the rate $r^{i}>0$. The utility $L_{i}$ could be equal to sale revenue, $L_{i}\left(u^{i}\right)=u^{i} p_{i}\left(u^{i}\right)$, where $p_{i}$ is an inverse demand function. The bequest function $S_{i}$ reflects the fact that the asset has externality effects over the players. The asset stock must satisfy $X(s) \geq 0$ almost surely (a.s.) for all $s$. Some of the assumptions listed below guarantee that both constraints $c^{i}(s) \geq 0$ and $X(s) \geq 0$ a.s. for all $s$ are satisfied in equilibrium. The asset evolves according to the SDE

$$
d X(s)=\left(F(X(s))-c^{i}-\sum_{j \neq i} \phi^{j}(s, X(s))\right) d s+\sigma(X(s)) d w(s),
$$

where $w$ is a standard Brownian motion defined on a complete probabilistic space $(\Omega, \mathcal{F}, \mathbf{P})$. We will denote by $\mathrm{E}_{t x}$ the conditional expectation with respect to the initial condition $(t, x)$ under the probability measure $\mathbf{P}$, where $x=X(t)>0$. The asset stock reproduces at the rate given by the production/recruitment function $F$, which may be a natural growth function describing the dynamics of a renewable resource, such as a fish population. In this case, it is common to consider $F$ with a maximum sustainable yield and with a maximum carrying capacity. We do not restrict ourselves to this case. The evolution of the asset is affected by stochastic fluctuations given by the diffusion term $\sigma(X(s))$. The uncertainty may come from inaccurate estimation of the resource reserves, which need to be continuously updated by the players. The game is widely used in the design of optimal harvest rules in stochastic fisheries under competition. Conrad (2012) uses this model to measure the effect of competition on the welfare of the players and carries out a calibration with real data and five players, finding that the predictions of the model are consistent with reality.

\subsection{The Euler-Lagrange equations}

The starting point of our approach to the game is the system of EL Eq. (2) given in Theorem 1. Let $\varphi_{i}=\left(L_{i}^{\prime}\right)^{-1} \circ S_{i}^{\prime}, i=1, \ldots, N$, that defines the optimal strategy of player $i$ at the end of the game. In the following lemma, the symbols $\partial_{t}, \partial_{x}$, denote total derivative with respect to $t, x$; details are given in Sect. 2 above.

Lemma 1 Suppose that the functions defining the game are twice continuously differentiable. Then a MPNE given by Markov strategies $\phi=\left(\phi^{1}, \ldots, \phi^{N}\right)$ of class $C^{1,2}$ satisfies the following system of EL equations:

$$
\begin{aligned}
r_{i} L_{i}^{\prime}\left(\phi^{i}(t, x)\right)= & \partial_{t} L_{i}^{\prime}\left(\phi^{i}(t, x)\right) \\
& +\partial_{x}\left(H^{i}\left(x, \phi(t, x), L_{i}^{\prime}\left(\phi^{i}(t, x)\right)\right)+\frac{1}{2} \sigma^{2}(x) \partial_{x} L_{i}^{\prime}\left(\phi^{i}(t, x)\right)\right)
\end{aligned}
$$

for $i=1, \ldots, N$, with final value

$$
\phi^{i}(T, x)=\varphi_{i}(x), \quad x>0
$$

and boundary condition

$$
\phi^{i}(t, 0)=0, \quad \forall t \leq T .
$$


Proof To obtain the EL equations, consider the current adjoint function and the current Hamiltonian of each player:

Adjoint function: $\Lambda^{i}\left(x,\left(c^{i} \mid c_{-i}\right)\right)=L_{i}^{\prime}\left(c^{i}\right)$,

Hamiltonian: $H^{i}\left(x,\left(c^{i} \mid c_{-i}\right), \lambda^{i}\right)=L_{i}\left(c^{i}\right)+\left(F(x)-c^{i}-\sum_{j \neq i} c^{j}(s)\right) \lambda^{i}$.

Now we apply Theorem 1 to obtain the EL Eq. (6) and final condition (7). The boundary condition (8) is a requirement imposed by feasibility.

In the infinite-horizon case, the EL equation system is still (6), but there is no terminal condition (7). For stationary Markov strategies the term $\partial_{t} L_{i}^{\prime}=L_{i}^{\prime \prime} \phi_{t}^{i}$ vanishes. Condition (8) becomes $\phi^{i}(0)=0$.

Let $R_{i}=-L_{i}^{\prime} / L_{i}^{\prime \prime}$ be the absolute risk tolerance index (the inverse of the absolute risk aversion index of Arrow-Prat) and $P_{i}=-L_{i}^{\prime \prime \prime} / L_{i}^{\prime \prime}$ be the absolute prudence index of player $i$ as defined in Kimball (1990). Taking total derivatives in (6) we get, for $i=1, \ldots, N$

$$
\begin{aligned}
& \phi_{t}^{i}(t, x)+\left(F(x)-\sum_{j=1}^{N} \phi^{j}(t, x)+\sigma^{\prime}(x) \sigma(x)\right) \phi_{x}^{i}(t, x)-\frac{1}{2} \sigma(x)^{2} P_{i}\left(\phi^{i}(t, x)\right)\left(\phi_{x}^{i}(t, x)\right)^{2} \\
& +\frac{1}{2} \sigma(x)^{2} \phi_{x x}^{i}(t, x)+R_{i}\left(\phi^{i}(t, x)\right)\left(r_{i}-F^{\prime}(x)+\sum_{j \neq i} \phi_{x}^{j}(t, x)\right)=0
\end{aligned}
$$

In the rest of the paper, we center on the symmetric game and on the symmetric $M P N E$. Thus, for all $i=1, \ldots, N$,

$$
L_{i}=L, \quad S_{i}=S, \quad r_{i}=r
$$

and hence we will drop the index in the functions defined above. The symmetry condition leads to the same risk tolerance and same prudence indexes for each player, $R_{i}=R$ and $P_{i}=P$ for any $i$, as well as the same terminal value at time $T, \varphi_{i}=\varphi$. Under this assumption, the symmetric MPNE leads, after rearrangement of (9), to the single EL equation

$$
\begin{aligned}
\phi_{t}(t, x)+ & \left(F(x)-N \phi(t, x)+(N-1) R(\phi(t, x))+\sigma^{\prime}(x) \sigma(x)\right) \phi_{x}(t, x) \\
& -\frac{1}{2} \sigma(x)^{2} P(\phi(t, x)) \phi_{x}(t, x)^{2}+\frac{1}{2} \sigma(x)^{2} \phi_{x x}(t, x)+R(\phi)\left(r-F^{\prime}(x)\right)=0,
\end{aligned}
$$

with final and boundary conditions

$$
\begin{aligned}
\phi(T, x) & =\varphi(x)=\left(L^{\prime}\right)^{-1}\left(S^{\prime}(x)\right), \quad x>0, \\
\phi(t, 0) & =0, \quad t<T,
\end{aligned}
$$

respectively. 
Let $\rho(c)=R(c) / c$ be the elasticity of intertemporal substitution for riskless consumption paths and $\pi(c)=P(c) c$ the relative prudence index.

\subsection{Assumptions}

To get our results of existence and uniqueness of a symmetric MPNE of the game, we need to impose several assumptions. They are justified after they are stated.

(A1) Functions $L, S, F$ and $\sigma$ are continuous in $[0, \infty)$, with $L(0)=F(0)=\sigma(0)=$ $0, \sigma(x)>0$ for $x>0$. Function $L$ is of class $C^{6}$ and $S, F$ and $\sigma$ are of class $C^{4}$ in $(0, \infty)$. Moreover, both $F^{\prime}$ and $\sigma^{\prime}$ are bounded in $(0, \infty)$.

(A2) The instantaneous utility function $L$ is strictly concave, with $L^{\prime \prime \prime} \geq 0$ and $R(0)=0$.

(A3) (a) There exist constants $0 \leq \rho^{-} \leq \rho^{+}<\frac{N}{N-1}$ such that

$$
\rho^{-} \leq \rho(c) \leq \rho^{+} \text {for all } c \geq 0
$$

(b) There exist constants $0 \leq \pi^{-} \leq \pi^{+}$such that

$$
\pi^{-} \leq \pi(c) \leq \pi^{+} \text {for all } c>0 \text {. }
$$

(A4) Function $\varphi$ satisfies $\varphi(0)=0$ and $\varphi(x)>0$ for $x>0$.

(A5) There is a function $f$, continuous in $[0, \infty)$ and of class $C^{4}$ in $(0, \infty)$ with $f^{\prime}$ bounded, satisfying $f(x)>0$ for $x>0$ such that

(a) $f^{\prime}\left(0^{+}\right)=\lim _{x \rightarrow 0^{+}} f(x) / x$ exists and is finite.

(b) The function $\varphi_{0}=\varphi / f$ satisfies

$$
M \equiv \sup _{x \in[0, \infty)} \varphi_{0}(x)<\infty, \quad m \equiv \inf _{x \in[0, \infty)} \varphi_{0}(x)>0 .
$$

(c) For $x>0$, let

$$
\begin{aligned}
& \gamma^{+}(x)=\max \left\{\rho^{-}\left(r-F^{\prime}(x)\right), \rho^{+}\left(r-F^{\prime}(x)\right)\right\}, \\
& \gamma^{-}(x)=\min \left\{\rho^{-}\left(r-F^{\prime}(x)\right), \rho^{+}\left(r-F^{\prime}(x)\right)\right\}
\end{aligned}
$$

and let

$$
\begin{aligned}
\beta^{+}(x)= & \left(F(x)+\sigma^{\prime}(x) \sigma(x)\right)\left(\frac{f^{\prime}(x)}{f(x)}\right)+\frac{1}{2} \sigma^{2}(x)\left(\frac{f^{\prime \prime}(x)}{f(x)}\right) \\
& -\frac{\pi^{-}}{2} \sigma^{2}(x)\left(\frac{f^{\prime}(x)}{f(x)}\right)^{2}+\gamma^{+}(x), \\
\beta^{-}(x)= & \left(F(x)+\sigma^{\prime}(x) \sigma(x)\right)\left(\frac{f^{\prime}(x)}{f(x)}\right)+\frac{1}{2} \sigma^{2}(x)\left(\frac{f^{\prime \prime}(x)}{f(x)}\right) \\
& -\frac{\pi^{+}}{2} \sigma^{2}(x)\left(\frac{f^{\prime}(x)}{f(x)}\right)^{2}+\gamma^{-}(x) .
\end{aligned}
$$


We assume that

$$
-\infty<\beta^{-} \equiv \inf _{x \in[0, \infty)} \beta^{-}(x), \quad \beta^{+} \equiv \sup _{x \in[0, \infty)} \beta^{+}(x)<\infty
$$

We will say that a function $f$ satisfying this assumption is a limiting function for the equilibrium.

Let us explain the assumptions.

- Assumption (A1) establishes the required smoothness of the data to apply our results and imposes finite marginal productivity at 0 and at $+\infty$. Concavity of $F$ is not needed. Note that (A1) implies the existence of constants $A$ and $\sigma$ such that $-A x \leq F(x) \leq A x$ and $0 \leq \sigma(x) \leq \sigma x$, for all $x \geq 0$. Hence, production functions with $F^{\prime}\left(0^{+}\right)=+\infty$ are excluded. This is the same assumption as in Foldes (2001).

- Assumption (A2) imposes strict concavity of the instantaneous utility $L$, a standard property that seems to be unavoidable for the existence of an interior and smooth equilibrium. The assumption $L^{\prime \prime \prime} \geq 0$ is typical in consumer theory and, for a concave utility $L$, it implies a positive prudence index. Consumers with a positive prudence index tend to make extra savings in the present date due to future income being random, a behavior known as precautionary savings. The condition $R(0)=0$ guarantees that the solution of the EL equation is non-negative.

- Assumption (A3) is key for proving existence of a smooth solution of the EL Eq. (10) and boundary conditions (11), and allows us to get convergence of the finitehorizon approximations to the solution of the infinite-horizon case with constant relative risk aversion instantaneous utility (CRRA henceforth). It assumes that both the elasticity of intertemporal substitution and the relative prudence index are bounded. However, we allow for unbounded utility functions $L$. In particular, the CRRA utility case is covered in our framework, as in the linear game defined below. Condition (a) means that the willingness of the players to substitute consumption across time is bounded by the ratio $N /(N-1)$, which depends on the number of players. As will be shown in Corollary 1 below, this condition cannot be relaxed if one seeks for interior and smooth equilibria, since it prevents the blow up of the solution of the EL Eq. (10) in finite time. The upper bound decreases as $N$ increases. In the limit when $N \rightarrow \infty$, it becomes 1 , but in the other extreme case with only one player, it places no constraint. Note that while we consider the stochastic game with $\rho(c)<N /(N-1)$, Dockner and Sorger (1996) studied the deterministic game with utility $L(c)=\sqrt{c}$ and two players, where $\rho(c)=$ $N /(N-1)=2$ for all $c$, leading to a continuum of symmetric discontinuous MPNE in the game with infinite horizon. Sorger (1998) attains the same result for more general utility functions satisfying $\rho(c) \geq N /(N-1)$. In both cases, the equilibrium exists due to the imposition of an upper bound in the consumption rate. The MPNE prescribes consumption at the maximal allowed rate for high enough values of the resource stock, so that the equilibrium is no longer interior. We study here the case left aside in the aforementioned references, in a stochastic 
environment.

- Assumption (A4) takes care of feasibility: it establishes that, at the final time $T$, consumption is positive as soon as there is something to consume, $\phi(T, x)>0$ if $x>0$, and that it is feasible, $\phi(T, 0)=0$.

- Assumption (A5) is a way to generalize our results including in our analysis games with the unbounded bequest function $S$, the production function $F$, and the diffusion coefficient $\sigma$. The selection of a given $f$ will depend on the form of these functions. Moreover, it provides lower and upper estimates for the consumption rule. The role of the constants $\beta^{+}$and $\beta^{-}$will be made clear in the proof of the theorem of the existence of smooth solutions of the EL equation, given in "Appendix."

We now introduce new notation that will be used throughout the paper

$$
\alpha^{+}=\rho^{+}(N-1)-N, \quad \alpha^{-}=\rho^{-}(N-1)-N \text {. }
$$

Assumption (A3) (a) implies that $\alpha^{+}<0$.

We illustrate the above assumptions in a particular game, that we will call the linear game with CRRA instantaneous utility, or simply the linear game, which is defined now for further reference.

Definition 4 The linear game corresponds to a CRRA instantaneous utility

$$
L(c)=\frac{c^{1-\delta}}{1-\delta}, \quad \text { if } 0<\delta<1,
$$

where both $F$ and $\sigma$ are linear, $F(x)=A x, \sigma(x)=\sigma x$, for some constants $A \geq$ $0, \sigma>0$.

Note that no specific functional form is imposed on the bequest function S. Linear production functions appear in endogenous growth models, where they are known as AK-models. We will use the linear game as a touchstone to illustrate the results obtained along the paper.

For the linear game, $f(x)=x$ is an adequate selection as a limiting function whenever the bequest value $S$ satisfies, in accordance with Assumption (A4) $S^{\prime}\left(0^{+}\right)=$ $\infty$ and $S^{\prime}(x)>0$ for $x>0$, and $\varphi(x)=S^{\prime}(x)^{-1 / \delta}$ satisfies Assumption (A5), that is

$$
\inf _{x>0} \frac{S^{\prime}(x)^{-1 / \delta}}{x}>0, \quad \sup _{x>0} \frac{S^{\prime}(x)^{-1 / \delta}}{x}<\infty .
$$

The remaining elements are $R(c)=\frac{c}{\delta}, P(c)=\frac{1+\delta}{c}, \rho(c)=\frac{1}{\delta}$ and $\pi(c)=1+\delta$, and the several constants defined above are

$$
\begin{aligned}
\rho & \equiv \rho^{-}=\rho^{+}=\frac{1}{\delta} \\
\pi & \equiv \pi^{-}=\pi^{+}=1+\delta \\
\alpha & \equiv \alpha^{+}=\alpha^{-}=(N-1) / \delta-N<0 \text { iff } \delta>1-1 / N
\end{aligned}
$$




$$
\beta \equiv \beta^{-}=\beta^{+}=A+\frac{\sigma^{2}}{2}(1-\delta)+\frac{r-A}{\delta} .
$$

\subsection{A class of utility functions satisfying the standing hypotheses}

There are many utility functions $L$ other than in the CRRA class that satisfy assumptions (A1-A3). We are specially interested in the class of utility functions with relative risk-seeking index given by

$$
\rho(c)=\frac{\psi_{1} c^{\theta}+\mu_{1}}{\psi_{2} c^{\theta}+\mu_{2}}, \quad \psi_{1}, \psi_{2}, \mu_{1}, \mu_{2}>0 .
$$

The parameter $\theta$ is arbitrary, but $\theta=0$ leads to a constant $\rho$ and thus, to the CRRA class. Our aim is to show a wider class of admissible utility functions that still have both $\rho$ and $\pi$ bounded. The case $\theta<0$ is a mirror case of $\theta>0$, exchanging the roles of the constants, since

$$
\rho(c)=\frac{\psi_{1}+\mu_{1} c^{-\theta}}{\psi_{2}+\mu_{2} c^{-\theta}}, \quad-\theta>0 .
$$

Hence, the results below are established only for $\theta>0$. Moreover, if $\Delta \equiv \psi_{1} \mu_{2}-$ $\psi_{2} \mu_{1}=0$, then $\rho=\mu_{1} / \mu_{2}$, leading to the CRRA class again, thus we assume $\Delta \neq 0$. It is clear that $\rho$ is bounded, since it is continuous in $[0, \infty)$ and $\lim _{c \rightarrow \infty} \rho(c)=\psi_{1} / \psi_{2}$ exists and is finite. In fact, the sign of $\rho^{\prime}$ is the sign of $\Delta$, thus $\rho$ is monotonous increasing or decreasing, and hence $\rho$ is bounded between the values $\mu_{1} / \mu_{2}$ and $\psi_{1} / \psi_{2}$. We will assume $\Delta>0$. Moreover, $\pi$ is also bounded. To show this claim, note that ${ }^{9} \rho^{\prime} c+\rho=-1+\rho \pi$, hence solving for $\pi$ one gets

$$
\pi(c)=1+\frac{1}{\rho(c)}+\frac{c \rho^{\prime}(c)}{\rho(c)} .
$$

The summand $1+\frac{1}{\rho(c)}$ is bounded. The other summand equals

$$
\frac{\theta \Delta c^{\theta}}{\left(\psi_{1} c^{\theta}+\mu_{1}\right)\left(\psi_{2} c^{\theta}+\mu_{2}\right)}
$$

which is continuous in $c$ and with limit $\frac{\theta \Delta}{\psi_{1} \psi_{2}}$ as $c \rightarrow \infty$. Thus, $\pi$ is bounded. In fact, the function $z \longmapsto \frac{z}{\left(\psi_{1} z+\mu_{1}\right)\left(\psi_{2} z+\mu_{2}\right)}$ attains a global maximum at $z^{*}=\sqrt{\frac{\mu_{1} \mu_{2}}{\psi_{1} \psi_{2}}}$. The explicit bounds for both $\rho$ and $\pi$, under the assumption $\Delta>0$, are

9 This relationship between $\rho$ and $\pi$ is general and follows from the identity $R^{\prime}=-1+R P$ and the definitions of $\rho$ and $\pi$. 


$$
\begin{aligned}
& \rho^{+}=\max \left\{\frac{\psi_{1}}{\psi_{2}}, \frac{\mu_{1}}{\mu_{2}}\right\}, \\
& \rho^{-}=\min \left\{\frac{\psi_{1}}{\psi_{2}}, \frac{\mu_{1}}{\mu_{2}}\right\}, \\
& \pi^{+}=1+\frac{1}{\rho^{-}}+\frac{\theta \Delta z^{*}}{\left(\psi_{1} z^{*}+\mu_{1}\right)\left(\psi_{2} z^{*}+\mu_{2}\right)}, \\
& \pi^{-}=1+\frac{1}{\rho^{+}} .
\end{aligned}
$$

In the following proposition, uniqueness means modulo multiplication and addition of constants.

Proposition 1 Let us assume that $\theta>0, \Delta>0$ and $\mu_{2}<\mu_{1}$. Then there exists a unique utility function $L$ that satisfies assumptions (A1-A3) and that has $\rho$ as given in (13). The marginal utility is given by

$$
L^{\prime}(c)=c^{-\frac{\mu_{2}}{\mu_{1}}}\left(\psi_{1} c^{\theta}+\mu_{1}\right)^{\frac{\Delta}{\psi_{1} \mu_{1} \theta}} .
$$

Proof By the comments and assumptions made before the proposition, both $\rho$ and $\pi$ are bounded. Note that $L^{\prime}$ defined in the proposition is infinitely differentiable in $(0, \infty)$ and positive. Let $L(c)=\int_{0^{+}}^{c} L^{\prime}(z) d z, c>0$. The definition is right, as this improper integral is convergent under the assumption $\mu_{2}<\mu_{1}$. This is because $\int_{0^{+}}^{1} c^{-\frac{\mu_{2}}{\mu_{1}}} d c$ is convergent and

$$
\lim _{c \rightarrow 0^{+}} c^{\frac{\mu_{2}}{\mu_{1}}} L^{\prime}(c)<\infty
$$

thus the comparison criterium leads to the claim. Moreover, $L(0)=0$ and $L$ is smooth in $(0, \infty)$. Starting from $(14)$ it is easy to calculate $L^{\prime \prime}$, that is negative (and thus $L$ strictly concave), as well as to check that $\rho$ is given by (13). The assumption $\Delta>0$ implies that $L^{\prime \prime \prime}>0$ as well.

We have not been able to find the explicit form of $L$. But to have an specific functional form is only for convenience, as the important characteristics of a consumer are given by the marginal utility and the attitude toward risk. They are the only pieces of information we need to proceed with our investigations.

It is also clear that if, for instance, we postulate an utility function $L$ with

$$
L^{\prime}(c)=c^{-\delta}\left(D c^{\theta}+E\right)^{\xi}
$$

and $\delta, \theta, \xi, D, E>0, \delta<1$, then the associated $\rho$ is of the form given in (13) after a suitable identification of the constants $\psi_{1}, \psi_{2}, \mu_{1}, \mu_{2}$ in terms of $\delta, D, E$, $\xi$. It is

$$
\begin{aligned}
& \psi_{1}=D, \\
& \mu_{1}=E,
\end{aligned}
$$




$$
\begin{aligned}
& \psi_{2}=D(\delta-\theta \xi), \\
& \mu_{2}=\delta E
\end{aligned}
$$

with $\theta \xi<\delta$ to ensure $\psi_{2}>0$. Also note that $\Delta=D E \theta \xi>0$. For this function $L, \rho^{+}=\frac{1}{\delta-\theta \xi}$ and $\alpha^{+}=\rho^{+}(N-1)-N<0$ holds for $N<\frac{1}{1-(\delta-\theta \xi)}$, where $\alpha^{+}$is defined in (12). This condition is fundamental in Theorem 2 below about existence of solutions of the PDE.

We will need for Sect. 5 a further characterization, that consists in isolating, within this class of utility functions, a subclass such that $\rho$ and $\pi$ show a linear tradeoff, $\pi=b-a \rho, a, b>0$. This means that increasing the elasticity of substitution one unit is only possible at the expense of decreasing the relative prudence index $a$ units.

Proposition 2 Let $\rho$ be as given in (13). Then, $\pi=b-a \rho$ if and only if

$$
\Delta=\psi_{1} \mu_{1} \theta
$$

and the constants $a, b$ are given by

$$
\begin{aligned}
& a=\frac{\psi_{2} \mu_{2}}{\psi_{1} \mu_{1}}, \\
& b=1+\frac{\psi_{2}}{\psi_{1}}+\frac{\mu_{2}}{\mu_{1}} .
\end{aligned}
$$

Proof Given $\rho$, we compute $L^{\prime}$ as in Proposition 1 and then $\pi$. The sufficient part is easily checked by substitution. For the necessary part, by imposing $\pi-b+a \rho=0$ for unknown positive constants $a, b$, three equations have to be fulfilled

$$
\begin{aligned}
& a\left(\frac{\psi_{1}}{\psi_{2}}\right)^{2}+(1-b)\left(\frac{\psi_{1}}{\psi_{2}}\right)+1=0, \\
& a\left(\frac{\mu_{1}}{\mu_{2}}\right)^{2}+(1-b)\left(\frac{\mu_{1}}{\mu_{2}}\right)+1=0, \\
& 2 a \psi_{1} \mu_{1}+(1-b)\left(\psi_{1} \mu_{2}+\mu_{1} \psi_{2}\right)+\Delta \theta+2 \psi_{2} \mu_{2}=0 .
\end{aligned}
$$

The two first equations say that the parabola $a \eta^{2}+(1-b) \eta+1$ has positive solutions $\psi_{1} / \psi_{2}$ and $\mu_{1} / \mu_{2}$. From this, one easily gets the sum and the product of roots

$$
\begin{aligned}
\frac{b-1}{a} & =\frac{\psi_{1}}{\psi_{2}}+\frac{\mu_{1}}{\mu_{2}}, \\
\frac{1}{a} & =\frac{\psi_{1}}{\psi_{2}} \frac{\mu_{1}}{\mu_{2}} .
\end{aligned}
$$

From the second equation, we get (17). Plugging this value into the first equation above we get (18). Now, using (19), and after some manipulations, the compatibility condition (16) arises. Finally, note that $b>1$ as well as $(1-b)^{2}-4 a=\left(\frac{\psi_{2}}{\psi_{1}}-\frac{\mu_{2}}{\mu_{1}}\right)^{2}>0$. 
Continuing with example (15), $L$ shows a linear relationship between $\rho$ and $\pi$ if and only if $\Delta=D E \theta \xi=D E \theta=\psi_{1} \mu_{1} \theta$, that is, $\xi=1$. Hence $L^{\prime}(c)=x^{-\delta}\left(D x^{\theta}+E\right)$ and then $L$ is the sum of two CRRA utilities.

We wish to establish the converse of Proposition 2. We need the following lemma, that will be also used in Sect. 5 below.

Lemma 2 Let the general Riccati differential equation $c \rho^{\prime}(c)=-a \rho^{2}+(b-1) \rho-1$, where $a, b>0$ are constants such $(b-1)^{2}>4 a$ and $b>1$. Then the general solution is

$$
\rho(c)=\frac{\eta K c^{\theta}+\frac{1}{\eta \theta}}{K c^{\theta}+\frac{a}{\theta}},
$$

where $\theta=2 a \eta-b+1>0, \eta>0$ is the bigger solution of $a \eta^{2}+(1-b) \eta+1=0$, and $K>0$ is arbitrary.

Proof According to Polyanin and Zaitsev (1995, eqn. 1.2.2.22), after taking $z=c^{b-1}$ and $y=\rho c^{1-b}$, the ODE transforms into the special Riccati equation $y^{\prime}(z)=\frac{a}{1-b} y^{2}+$ $\frac{1}{1-b} z^{-2}$. The general solution of this equation is, again following Polyanin and Zaitsev (1995, eqn. 1.2.2.36)

$$
y(z)=\frac{\eta}{z}-\frac{z^{2 \frac{a}{1-b} \eta}}{\frac{\frac{a}{1-b} z}{2 \frac{a}{1-b} \eta+1} z^{2 \frac{a}{1-b} \eta}+K},
$$

where $K$ is an arbitrary constant and $\eta$ is solution of the quadratic equation $a \eta^{2}+$ $(1-b) \eta+1=0$. Now, turning back to the original variables, we obtain

$$
\rho(c)=\eta-\frac{c^{-2 a \eta+b-1}}{\frac{\frac{a}{1-b}}{2 \frac{a}{1-b} \eta+1} c^{-2 a \eta+b-1}+K} .
$$

Manipulating this expression, and using the definition of $\theta$ and of $\eta$, it is straightforward to get (20). Finally, the assumptions done imply that $\eta>0$ exists and that $\theta>0$, since

- The quadratic equation admits the positive solution $\eta=(b-1) / 2 a+$ $\sqrt{(b-1)^{2}-4 a} / 2 a$.

$-\theta>0$ is equivalent to $\eta>(b-1) / 2 a$, which is true by the item above.

Looking at (13) and at (20), the following identification of parameters follows

$$
\begin{aligned}
\theta & =2 a \eta-b+1, \\
\psi_{1} & =\eta K, \\
\mu_{1} & =\frac{1}{\eta \theta}, \\
\psi_{2} & =K, \\
\mu_{2} & =\frac{a}{\theta} .
\end{aligned}
$$

Of course, (16), (17) and (18) hold. 
Proposition 3 Let $L$ be a three-time differentiable utility function such that $\pi=b-a \rho$ for some constants $a, b>0$, where $b>1$ and $(b-1)^{2}>4 a$. Let $\eta$ be the larger solution of the quadratic equation $a \eta^{2}+(1-b) \eta+1=0$. Then associated $\rho$ is given by (13), with parameters given in (21). If $L(0)=0$, then a $\rangle<1$ holds.

Proof It is consequence of the previous lemma. All the parameters are positive and $\Delta=\frac{K}{\eta \theta}\left(a \eta^{2}-1\right)=K>0$ by assumption, since $\eta \theta=a \eta^{2}-1$, by definition of $\eta$ and $\theta$. When $L(0)=0$, the improper integral $\int_{0}^{c} L^{\prime}(z) d z$ is convergent, see the proof of Proposition 1. This implies that $\mu_{2}<\mu_{1}$, which is $a \eta<1$.

\section{Existence of a symmetric MPNE}

In the following results, we will suppose that the assumptions imposed in the above section hold. To show existence of an MPNE, we proceed in two steps. First, we establish a result about the existence of a solution to the EL equation, providing at the same time upper and lower bounds for the solution. In a second step, we will prove that under suitable additional conditions, the solution qualifies as an MPNE of the symmetric game. The consideration of a limiting function $f$ in the estimates found for the solution of the EL equation in the next result allows us to drop the boundedness hypothesis that is commonly assumed in the PDE literature. Boundedness is a severe limiting assumption in economic models, since it eliminates CRRA utility functions and linear production functions from the analysis, even though they are by far the more popular and widely used in applications. Our approach will be useful in proving that solutions of the EL equation are in fact an MPNE of the game for a wide range of utility and production functions, as well as in analyzing the turnpike properties of the finite-horizon MPNE.

\subsection{Existence of smooth solutions to the Euler-Lagrange equation}

The proof of the theorem below uses classical results of existence of solution to PDEs, adapted to our framework with unbounded functions and unbounded state space. For this reason (unboundedness), those results cannot be applied directly, so we combine them with an approach that uses the maximum and minimum values of solutions existing in finite time and bounded intervals of the state space. These solutions provide upper and lower estimates for the solution that prevent the blow up or that the solution could become zero or negative, in terms of the limiting function $f$ defined in (A5) above. Thus, besides proving existence of solution, we find a "window" moving with $t$ where the solution is confined for any $x$. As we will see, in the CRRA case, the window stretches as the final date $T$ tends to infinity, so that the finite-horizon solution converges to a solution of the infinite-horizon EL equation. To find the upper and lower bounds, we use a maximum principle for nonlinear parabolic PDEs together with the well-known envelope theorem due to Danskin (1966).

Recall the definitions made in the previous section of the constants $\alpha^{+}, \alpha^{-}$in (12), as well as of $M=\sup _{x \in(0, \infty)} \frac{\varphi(x)}{f(x)}, m=\inf _{x \in(0, \infty)} \frac{\varphi(x)}{f(x)}$ and $\beta^{+}, \beta^{-}$in (A5). We will also use the following functions of $t$ 


$$
\begin{aligned}
& k_{-}(t)=\frac{m \beta^{-} e^{\beta^{-}(T-t)}}{\alpha^{-} m\left(\sup _{(0, \infty)} f^{\prime}\right)\left(1-e^{\beta^{-}(T-t)}\right)+\beta^{-}}, \\
& k_{+}(t)=\frac{M \beta^{+} e^{\beta^{+}(T-t)}}{\alpha^{+} M\left(\inf _{(0, \infty)} f^{\prime}\right)\left(1-e^{\beta^{+}(T-t)}\right)+\beta^{+}} .
\end{aligned}
$$

Note that thanks to our assumptions, these functions are well defined and are obviously smooth, with $0<k_{-}(t)<k_{+}(t)<\infty$ for all $t \leq T$. In the following result, uniqueness has to be understood in the class of functions considered in the theorem, satisfying the given bounds.

Theorem 2 Let assumptions (A1-A5) hold, with limiting function $f$. Then there is a unique non-negative solution $\phi$ of class $C^{2,4}$ of the Cauchy problem (10) that satisfies $\phi(t, 0)=0$ for all $t \in[0, T]$ and the estimates

$$
0<k_{-}(t) f(x) \leq \phi(t, x) \leq k_{+}(t) f(x)
$$

for all $x>0$ and $t \in[0, T]$.

Proof See "Appendix."

\subsection{A nonexistence result}

The lower estimate provided in Theorem 2 is useful to give a negative criterion for the existence of an interior MPNE. To fix ideas, we consider a CRRA utility $L(c)=$ $c^{1-\delta} /(1-\delta)$ with $\rho(c)>N /(N-1)$ (equivalently, $\left.\alpha=(N-1) / \delta-N>0\right)$. The same analysis can be done for a general utility function, using the lower bound $\alpha^{-}$defined in the previous section. As the following result shows, the (local) smooth solution of the EL equation explodes in finite time, so no global smooth solution exists. Since the EL equation is necessary for the optimality of a smooth MPNE, we can conclude that no equilibrium exists in this case. The intuition for this behavior is as follows: the willingness of the players to substitute consumption across time is too high and this motivates a strong competition to obtain the resource. Eventually, the consumption rate blows up in finite time. As in the discrete time model game studied by Dutta and Sundaram (1993b), the Markovian first-best solution $(N=1)$ always exist, but in the competition version, the existence is not guaranteed, unless an upper bound is imposed in the intertemporal rate of substitution of the players, bound that is related with the number of players.

Corollary 1 Suppose that in the conditions of Theorem 2, $\alpha>0$. Then, for T large enough, there is no smooth solution of the EL equation.

Proof We reason by contradiction, assuming that a smooth solution exists. The bounds we have found in the proof of Theorem 2 above are still valid. In particular, the lower bound with $\alpha>0$ implies that the denominator of $k_{-}(t)$ in (22) vanishes at time

$$
\widehat{t}=T-\frac{1}{\beta} \ln \left(1+\frac{\beta}{\alpha m \sup _{x \in(0, \infty)} f^{\prime}(x)}\right),
$$


with $0<\widehat{t}<T$, hence if a solution exists, it would satisfy

$$
\phi(t, x) \geq k_{-}(t) f(x) \rightarrow \infty \quad \text { as } t \rightarrow \widehat{t}^{+},
$$

thus, the solution becomes infinite at a finite instant of time, reaching a contradiction.

The corollary shows an extreme sensitivity of the MPNE with respect to variations in the number of players that have free access to the asset, as explained in the corollary just above. In fact, it implies the nonexistence of an interior MPNE, and thus, the imposition of an upper bound in consumption is needed for the equilibrium to exist. In the CRRA case, with $\delta$ denoting the elasticity of the marginal utility, there is a critical number, $\hat{N}=$ integer part of $(1-\delta)^{-1}$, such that if the number of players is $\hat{N}$ and a new player enters the game, then the game changes drastically, since the MPNE blows up in finite time. For instance, if $\delta<1 / 2$, then $\hat{N}=1$, so the solution in the single player case is interior (as is always the case for the one-player model under our assumptions), but when a new identical player enters the game, so that the game becomes a duopoly, the competition is so intense that the players would like to consume all the resource instantaneously, a characteristic already revealed by Reinganum and Stokey (1985) in a related game. Dockner and Sorger (1996) and Sorger (1998) handle this case in the deterministic game by imposing an upper bound in the maximal consumption rate, proving the existence of a continuum of discontinuous MPNE.

\subsection{Markov Perfect Nash Equilibrium and value function}

We next show that the solution of the EL equation is indeed an MPNE of the game. Given $\phi(t, x)$ a solution of the EL Eq. (10) satisfying the boundary conditions (11), let us define for $x>0$

$$
\lambda(t, x)=\Lambda(x, \phi(t, x))=L^{\prime}(\phi(t, x))
$$

and let $H(x, c, p)=L(c)+p(F(x)-N c)$. We will prove that $\lambda$ is the costate variable or asset shadow price, that is $V_{x}=\lambda$, where $V$ denotes the value function of the symmetric game. We will also show that $\phi$ is an MPNE and we will provide the following expression for the value function in terms of $\phi(t, x)$ : for $x>0$

$$
\begin{aligned}
V(t, x)= & \int_{\ell}^{x} \lambda(t, z) d z+\int_{t}^{T} e^{-r(s-t)}\left(H(\ell, \phi(s, \ell), \lambda(s, \ell))+\frac{1}{2} \sigma^{2}(\ell) \lambda_{x}(s, \ell)\right) d s \\
& +e^{-r(T-t)} S(\ell)
\end{aligned}
$$

where $0<\ell<x$ is an arbitrary constant, and $V(t, 0)=0$ for any $t$. The corresponding expression for the value function in the infinite-horizon case is given below the theorem.

In the proof of the following theorem, we will need to define constants $k_{-}$and $k_{+}$, that satisfy the inequalities 


$$
\begin{aligned}
0 & <k_{-} \equiv \min \left\{m,-\frac{\beta^{-}}{\alpha^{-} \sup _{(0, \infty)} f^{\prime}}\right\} \leq k_{-}(t) \leq k_{+}(t) \\
& \leq \max \left\{M,-\frac{\beta^{+}}{\alpha^{+} \inf _{(0, \infty)} f^{\prime}}\right\} \equiv k_{+},
\end{aligned}
$$

for any $0 \leq t \leq T$, since $\alpha^{+}<0$.

Theorem 3 Assume that the conditions of Theorem 2 hold with a limiting function $f$. Let $0<\gamma<1$ be a constant such that for any $k>0$, there is a polynomial $q$ and $a$ constant $D>0$ such that

$$
\begin{aligned}
& \forall x>0, \quad\left|L^{\prime}(k f(x))\right| \leq q(x)+D x^{-\gamma}, \\
& \lim _{x \rightarrow 0^{+}} x^{-\gamma} F(x)=0
\end{aligned}
$$

and

$$
\lim _{x \rightarrow 0^{+}} \sigma(x)^{2} L^{\prime \prime}(f(x))=0 .
$$

Then, the non-negative solution $\phi$, whose existence is guaranteed by Theorem 2 , is an MPNE of the differential game. Moreover, the value function of the players, given by (23), is of class $C^{2,5}$ in $(0, \infty)$, continuous in $[0, \infty)$ and $V(t, 0)=0$ for all $t \geq 0$.

Proof We first claim that for any $T$ there exists a unique strong solution of

$d X(s)=(F(X(s))-N \phi(s, X(s))) d s+\sigma(X(s)) d w(s), \quad t<s \leq T, \quad X(t)=x$,

which is positive almost surely. To show this, observe that both $F$ and $\sigma$ are locally Lipschitz by assumption. On the other hand, the monotone condition

$$
x F(x)-N x \phi(t, x)+\frac{1}{2} \sigma^{2}(x) \leq K x^{2}
$$

holds for some constant $K$, since $\phi(t, x) \geq 0, x \geq 0$ and because by (A.1) $F(x) \leq A x$ and $\sigma(x) \leq \sigma x$ for some constants $A$ and $\sigma$. Thus, according to Theorem 3.6 in Mao (1997), there exists a unique strong solution of (27). Moreover, since $F(0)=\sigma(0)=$ $\phi(t, 0)=0$, Lemma 3.2 in Mao (1997) implies $X \geq 0$ a.s.

To continue with the proof, let $W(t, x)$ be the right-hand side of (23). From the regularity of $\phi, W$ is of class $C^{2,5}$ in $[0, T) \times(0, \infty)$. Let us show that $\lim _{x \rightarrow 0^{+}} W(t, x)=0$, so that $W(t, 0)$ can be defined as $0=V(t, 0)$. Note that by $(24)$ and since $L^{\prime}$ is decreasing and $k_{-} f(x) \leq \phi(t, x) \leq k_{+} f(x)$ for all $x>0$

$$
|\lambda(t, x)|=\left|L^{\prime}(\phi(t, x))\right| \leq \max \left\{\left|L^{\prime}\left(k_{-} f(x)\right)\right|,\left|L^{\prime}\left(k_{+} f(x)\right)\right|\right\} \leq q(x)+D x^{-\gamma},
$$

for a suitable polynomial $q$ and constant $D$. Hence

$$
\int_{0}^{x}|\lambda(t, z)| d z \leq \int_{0}^{x} q(z) d z+D \frac{x^{1-\gamma}}{1-\gamma} \rightarrow 0, \quad \text { as } x \rightarrow 0^{+},
$$


since the integral in the right-hand side is an homogeneous polynomial and $\gamma<1$. Since $\int_{0}^{x} z^{-\gamma} d z$ is convergent, then $\int_{\ell}^{x} \lambda(t, z) d z$ tends to zero as $x \rightarrow 0^{+}$(and thus $\ell \rightarrow 0^{+}$), which is the first integral in the definition of $W$. The second integral defining $W$ is with respect to time. Regarding the Hamiltonian $H$, note that $L(0)=0$, so by concavity of $L, L^{\prime}(c) c \leq L(c)$. Hence

$$
\begin{aligned}
L^{\prime}(\phi(t, \ell))|F(\ell)-N \phi(t, \ell)| & \leq L^{\prime}(\phi(t, \ell)) F(\ell)+N L^{\prime}(\phi(t, \ell)) \phi(t, \ell) \\
& \leq\left(q(\ell)+D \ell^{-\gamma}\right) F(\ell)+N L(\phi(t, \ell))
\end{aligned}
$$

tends to 0 uniformly since $\ell \rightarrow 0^{+}$by $(25)$ and $\phi(t, \ell) \rightarrow 0$. The second summand in the integral is $\frac{1}{2} \sigma^{2}(\ell) \lambda_{x}(s, \ell)$. Let us show that it tends to 0 as $\ell \rightarrow 0$. Note that $\phi(t, 0)=0$ and

$$
k_{-} \frac{f(x)}{x} \leq \frac{\phi(t, x)}{x} \leq k_{+} \frac{f(x)}{x}
$$

imply $k_{-} f^{\prime}\left(0^{+}\right) \leq \phi_{x}\left(t, 0^{+}\right) \leq k_{+} f^{\prime}\left(0^{+}\right)$, thus $\phi_{x}(t, \cdot)$ is bounded around 0 and in consequence

$$
\sigma^{2}(\ell) L^{\prime \prime}\left(k_{+} f(\ell)\right) \phi_{x}(s, \ell) \geq \sigma^{2}(\ell) \lambda_{x}(s, \ell) \geq \sigma^{2}(\ell) L^{\prime \prime}\left(k_{-} f(\ell)\right) \phi_{x}(s, \ell)
$$

where we have used that $L^{\prime \prime \prime} \geq 0$. Now, by (26), both sides tend to 0 as $\ell \rightarrow 0$.

Now let us show that $W$ satisfies the Hamilton-Jacobi-Bellman equation for any $x>0$ and $t<T$. Observe that, for $x>0, W_{x}=\lambda$ and $W_{x x}=\lambda_{x}$ by the definition of $W$. Let $g(t)$ be the function of $t$ given in the second summand of (23). The derivative is

$$
g^{\prime}(t)=r g(t)+H(\ell, \phi(t, \ell), \lambda(t, \ell))+\frac{1}{2} \sigma^{2}(\ell) \lambda_{x}(t, \ell) .
$$

Also notice that

$$
W_{t}(t, x)=\int_{\ell}^{x} \lambda_{t}(t, z) d z+g^{\prime}(t)
$$

Now, integrating with respect to $x$ in (6), recalling the definition of $W$ and rewriting in terms of $W_{x}, W_{x x}$ we have

$$
\begin{aligned}
0= & -r W(t, x)+r g(t)+W_{t}(t, x)-g^{\prime}(t)+H(x, \phi(t, x), \lambda(t, x)) \\
& -H(l, \phi(t, l), \lambda(t, l))+\frac{1}{2} \sigma^{2}(x) \lambda_{x}(t, x)-\frac{1}{2} \sigma^{2}(l) \lambda_{x}(t, l) \\
= & -r W(t, x)+W_{t}(t, x)+H(x, \phi(t, x), \lambda(t, x))+\frac{1}{2} \sigma^{2}(x) \lambda_{x}(t, x) \\
= & -r W(t, x)+W_{t}(t, x)+H\left(x, \phi(t, x), W_{x}(t, x)\right)+\frac{1}{2} \sigma^{2}(x) W_{x x}(t, x) .
\end{aligned}
$$


Given Hypothesis (A2), the function $c^{i} \longmapsto H\left(x,\left(c^{i} \mid c_{-i}\right), \lambda\right)$ is concave and, by the definition of $\lambda$,

$$
H_{c^{i}}\left(t, x,\left(c^{i} \mid c_{-i}\right), \lambda\right)=H_{c^{i}}\left(t, x,\left(c^{i} \mid c_{-i}\right), L^{\prime}\left(c^{i}\right)\right)=L^{\prime}\left(c^{i}\right)-L^{\prime}\left(c^{i}\right)=0 .
$$

Since critical points of concave functions are global maximum, we get that for arbitrary admissible consumption strategies $c^{i}$ and for $i=1, \ldots, N$

$$
\begin{aligned}
0 & =-r W(t, x)+W_{t}(t, x)+h\left(x, \phi, W_{x}(t, x)\right)+\frac{1}{2} \sigma^{2}(x) W_{x x}(t, x) \\
& \geq-r W(t, x)+W_{t}(t, x)+H\left(t, x,\left(c^{i} \mid \phi_{-i}\right), W_{x}(t, x)\right)+\frac{1}{2} \sigma^{2}(x) W_{x x}(t, x) .
\end{aligned}
$$

Hence, $W$ is a solution of the HJB Eq. (4) given in Sect. 2. Moreover, $W(T, x)=S(x)$. This is easily seen as follows

$$
\begin{aligned}
W(T, x) & =\int_{\ell}^{x} \lambda(T, z) d z+S(\ell)=\int_{\ell}^{x} L^{\prime}(\phi(T, z)) d z+S(\ell) \\
& =\int_{\ell}^{x} L^{\prime}(\varphi(z)) d z+S(\ell)=S(x)
\end{aligned}
$$

since $\varphi=\left(L^{\prime}\right)^{-1} \circ S^{\prime}$. Now, condition (28), identity $V_{x}=\lambda$, together with the continuity of $W(t, x)$ at $x=0$ proved above, imply that $W$ is indeed the value function of the problem and $\phi^{i}$ is the MPNE of player $i, i=1, \ldots, N$. This is because $V$ is polynomially bounded in $x$ by (24), the definition of $V$ given in (23) as a primitive with respect to $x$ of $L^{\prime}(\phi(t, x))$, and the bounds found for $\phi$, so we can apply the verification theorems of Dockner et al. (2000) or Fleming and Soner (2006).

Remark 1 The result is extended to the infinite-horizon game with the help of the usual transversality condition. We analyze only the stationary case. From (10), the stationary MPNE $\phi$ is characterized by the EL equation

$$
\begin{aligned}
& \left(F(x)-N \phi(x)+(N-1) R(\phi(x))+\sigma^{\prime}(x) \sigma(x)\right) \phi^{\prime}(x)-\frac{1}{2} \sigma(x)^{2} P(\phi(x)) \phi^{\prime}(x)^{2} \\
& +\frac{1}{2} \sigma(x)^{2} \phi^{\prime \prime}(x)+R(\phi(x))\left(r-F^{\prime}(x)\right)=0 .
\end{aligned}
$$

Now, no terminal condition is imposed, but we still have to consider the boundary condition $\phi(0)=0$. The value function can be expressed in terms of the MPNE as

$$
\begin{aligned}
V(x)= & \int_{\ell}^{x} L^{\prime}(\phi(z)) d z+\frac{1}{r}\left(L(\phi(\ell))+L^{\prime}(\phi(\ell))(F(\ell)-N \phi(\ell))\right. \\
& \left.+\frac{1}{2} \sigma^{2}(\ell) L^{\prime \prime}(\phi(\ell)) \phi^{\prime}(\ell)\right)
\end{aligned}
$$

where $0<\ell<x$ is a fixed, arbitrary constant. 
In the following result, $X^{c}$ denotes the solution of (5) when the admissible profile of strategies $(c, \ldots, c)$ is played.

Theorem 4 Assume that the conditions of Theorem 3 hold and that for all $c \in \mathcal{U}$, the transversality condition

$$
\limsup _{T \rightarrow \infty} \mathrm{E}_{t x}\left\{e^{-r(T-t)} V\left(X^{c}(T)\right)\right\}=0
$$

holds. Then, a stationary and smooth solution $\phi$ of the Cauchy problem (29) is an MPNE of the differential game. Moreover, the value function of the players is given by (30).

Proof We can follow the same arguments as for the finite-horizon case shown in Theorem 3 above, by selecting a bounded function $S$ fulfilling the hypotheses required by that theorem, so that $\lim _{T \rightarrow \infty} e^{-r T} S(x)=0$. For a Markov stationary strategy $\phi(x)$, the costate variable $\lambda$ is also independent of time. Taking the limit as $T \rightarrow \infty$ in the function $g$ obtained from (23) in the proof of Theorem 3, we have

$$
\begin{aligned}
g(t, \ell) & =\lim _{T \rightarrow \infty} \int_{t}^{T} e^{-r(s-t)}\left(H(\ell, \phi(s, \ell), \lambda(s, \ell))+\frac{1}{2} \sigma(\ell)^{2} \lambda_{x}(s, \ell)\right) d s \\
& =\left(H\left(\ell, \phi(\ell), L^{\prime}(\phi(\ell))\right)+\frac{1}{2} \sigma(\ell)^{2} L^{\prime \prime}(\phi(\ell)) \phi^{\prime}(\ell)\right) \lim _{T \rightarrow \infty} \int_{t}^{T} e^{-r(s-t)} d s \\
& =\frac{1}{r}\left(H\left(\ell, \phi(\ell), L^{\prime}(\phi(\ell))\right)+\frac{1}{2} \sigma(\ell)^{2} L^{\prime \prime}(\phi(\ell)) \phi^{\prime}(\ell)\right) .
\end{aligned}
$$

We need the transversality condition of the theorem to apply a verification theorem of Dockner et al. (2000) or Fleming and Soner (2006) for the infinite-horizon case.

\section{The stochastic Keynes-Ramsey Rule}

In this section, we describe how the EL Eq. (10) defines a stochastic KR rule for the MPNE. This has the form of a forward-backward stochastic differential equation (FBSDE). ${ }^{10}$ Note that the representation depends on the forward process chosen. In this section we consider the stock process in equilibrium, $X$, but in further sections, we will change to another processes which are more useful to our purposes.

\footnotetext{
10 FBSDEs were introduced by Bismut (1973) for stochastic control problems and studied with more generality in Pardoux and Peng (1990). FBSDEs play a central role in the statement of the stochastic maximum principle, see Peng (1990), Yong and Zhou (1999), or Ma and Yong (1999). FBSDEs in optimization models play the same role as the Hamiltonian system for the state-costate variable in deterministic dynamics, that is, they constitute a part of the necessary conditions for optimality. In our game, the forward part corresponds to the new state $Y$ and the backward part to the optimal strategy $C$. The definition of a backward SDE is not straightforward, as the filtration of the Brownian motion is an increasing family of $\sigma$-algebras, but the stochastic process $C$ evolves in the opposite direction of time. The measurability problems are overcome by introducing the process $Z$, which is an integral part of the definition of the solution.
} 
Given a solution $\phi(t, x)$ of the PDE (10) satisfying $\phi(T, x)=\varphi(x)$, let $C(s)=$ $\phi(s, X(s))$ for $s \geq t$, where $X$ satisfies the SDE of the stock variable in equilibrium

$$
d X(s)=(F(X(s))-N C(s)) d s+\sigma(X(s)) d w(s), \quad X(t)=x>0,
$$

A solution of the stochastic $\mathrm{KR}$ rule that we will show below is a triplet $(Y, C, Z)$ of $\left\{\mathcal{F}_{S}\right\}_{s \geq t}$-adapted processes.

Theorem 5 Assume that the hypotheses of Theorem 3 hold and that $\sigma^{\prime \prime}$ is bounded. Then, the symmetric MPNE is described by the stochastic KR rule given by the forward $S D E$ (31) and the backward SDE

$$
\begin{aligned}
\frac{d C(s)}{C(s)}= & \left(\rho(C(s))\left(F^{\prime}(X(s))-r\right)+(1-N) \rho(C(s)) C(s) \frac{Z(s)}{\sigma(X(s))}-\sigma^{\prime}(X(s)) Z(s)\right. \\
& \left.+\frac{1}{2} \pi(C(s)) Z^{2}(s)\right) d s+Z(s) d w(s)
\end{aligned}
$$

with final value

$$
C(T)=\varphi(X(T)),
$$

where $Z$ is a square-integrable $\left\{\mathcal{F}_{S}\right\}_{s \geq t}$-adapted process. Moreover, $Z$ is non-negative a.s. in the case that both $F$ and $S$ are concave.

Proof Under the assumptions and by Theorem 2, there is a unique non-negative classical solution $\phi(t, x)$ of Eq. (10) satisfying $\phi(T, x)=\varphi(x)$ and $\phi(s, 0)=0$ for any $s \geq t$. Consider the forward SDE (31). Since $\phi, F$ and $\sigma$ are uniformly Lipschitz, by Lemma 3.2 in Mao (1997), the SDE admits a unique strong solution $X(s), s \geq t$ for all $t \geq 0$, which satisfies $X(s)>0$ a.s.

By Itô's formula applied to $C(s)=\phi(s, X(s))$ and using the EL Eq. (10), we get (we omit the argument $(s, X(s))$ on $\phi$ and its derivatives, to simplify notation)

$$
\begin{aligned}
d C(s)= & \left(\phi_{t}+(F(X(s))-N \phi) \phi_{x}+\frac{1}{2} \sigma^{2}(X(s)) \phi_{x x}\right) d s+\phi_{x} \sigma(X(s)) d \omega(s) \\
= & \left(R(\phi)\left(F^{\prime}(X(s))-r\right)+(1-N) R(\phi) \phi_{x}-\sigma^{\prime}(X(s)) \sigma(X(s)) \phi_{x}\right. \\
& \left.+\frac{1}{2} \sigma^{2}(X(s)) P(\phi) \phi_{x}^{2}\right) d s+\phi_{x} \sigma(X(s)) d \omega(s)
\end{aligned}
$$

Defining $Z(s)=\sigma(X(s)) \phi_{x}(s, X(s)) / \phi(s, X(s))$ for $s \geq t \geq 0$, and recalling that $R=c \rho, P=\pi / c$, the above equality reduces to (32). Note that $Z$ is square-integrable and $\left\{\mathcal{F}_{s}\right\}_{s \geq t}$-adapted by the regularity of the functions involved. When $T<\infty$ the terminal condition for $C$ at $T$ comes from (11). In the case of an infinite horizon, we follow the definition given in Ma and Yong (1999), considering finite-horizon approximations where the terminal condition for $C$ is given by selecting previously a suitable bequest function $S$, as explained in the sections above. Finally, since by Theorem 8 (below) $\phi_{x} \geq 0$, we have $Z(s)=\sigma(X(s)) \phi_{x}(s, X(s)) / \phi(s, X(s)) \geq 0$ a.s. 
Equality (32) shows how consumption of each player is distributed in equilibrium across time. The expected rate of growth in future consumption with respect to current consumption is, for $s \geq t, t \geq 0$

$$
E_{s}\left(\frac{d C}{C}\right)=\left(\rho(C)\left(F^{\prime}(X)-r\right)+(1-N) \rho(C) C \frac{Z}{\sigma(X)}+\frac{1}{2} \pi(C) Z^{2}-\sigma^{\prime}(X) Z\right) d s .
$$

Due to the presence of uncertainty, the rule is much more complex than in the deterministic case. ${ }^{11}$ Three additional summands appear, which depend on $Z$ and collect the uncertainty effects. The second term, $(1-N) \rho(C) C \frac{Z}{\sigma}$, shows how the number of players and the uncertainty interact; this summand is negative when there are more than one player, and is larger in absolute value as the number of players increases. The third summand, $\frac{1}{2} \pi(C) Z^{2}$, contains the prudence attitude of the player. Since we are supposing that $\pi>0$, uncertainty speeds up mean consumption in comparison with the deterministic case. More prudent agents prefer to plan higher mean increments in consumption than less prudent ones. The fourth and last term, $-\sigma^{\prime}(X) Z$, is sensitive to the variation of instantaneous variance, $\sigma^{\prime}$.

The backward-forward pair of Eqs. (31) and (32) is useful is exploring inverse problems, as that studied in Chang (1988) for the stochastic optimal growth model. That is, given a consumption function, the issue is to determine utility and recruitment/production functions that rationalize the consumption function as optimal in the control problem.

We address here a related question: to determine the set of admissible utilities for which the MPNE is linear in the infinite horizon game with linear production and variance functions. As it is well known, CRRA utilities are in this set. Are there any more? It comes as a surprise that the answer is positive. To show this, let $F(x)=A x$ and $\sigma(x)=\sigma x$, where the constants are positive. Suppose that the MPNE is $\phi(x)=$ $\mu x$, with $\mu>0$. Note that $C(s)=\mu X(s)$, thus $d C=\mu d X=\mu(A X-N C) d s+$ $\mu \sigma X d w$, or writing this equation in terms of $C, d C=(A-\mu N) C d s+\sigma C d w$. Thus, consumption is a geometric Brownian motion along the equilibrium trajectory. In consequence, the expected mean increment of consumption, which is given in (34), is constant, equal to $\epsilon \equiv A-\mu N$. Let us compute this term. In the proof of Theorem 5, $Z$ was defined as $Z=\sigma(X) \phi^{\prime}(X) / \phi(X)$. In this case, we have $Z=\sigma$, constant. Then, from (34) and the fact that $C$ is a geometric Brownian motion, we obtain the identity

$$
(A-r) \rho(c)+(1-N) \mu \rho(c)+\frac{1}{2} \sigma^{2} \pi(c)-\sigma^{2}=\epsilon .
$$

\footnotetext{
11 The deterministic KR rule or "modified Ramsey rule" is obtained setting $N=1$ and $\sigma \equiv 0$ in (33), to get

$$
\frac{\dot{C}}{C}=\rho(C)\left(F^{\prime}(X)-r\right)
$$
}

where the (deterministic) process $X$ satisfies

$$
\dot{X}=F(X)-N C \text {. }
$$


This gives a relationship between $\rho$ and $\pi, \pi=b-a \rho$, where

$$
a=\frac{2}{\sigma^{2}}(A-r+(1-N) \mu), \quad b=2 \frac{\epsilon+\sigma^{2}}{\sigma^{2}} .
$$

Assume that $a>0, b>1$ and $(b-1)^{2}>4 a$. The inequality $a>0$ is true for the one-player case when $A>r$, but it also holds for $N>1$ if $A$ is large enough. Hence we can establish the following theorem.

Theorem 6 Consider the infinite-horizon game with linear recruitment and instantaneous variance and with $a$ and $b$ given in (35) that satisfy $0<a<1, b>1$ and $(b-1)^{2}>4 a$. Then there exists utility functions with non-constant elasticity of intertemporal substitution $\rho$ and discount factors $r>0$, such that the linear rule, $\phi(x)=\mu x$, is a MPNE of the game.

Proof To find $L$ supporting the MPNE, we first use Lemma 2 that gives the solution of the equation $c \rho^{\prime}+\rho=-1+\rho \pi$ with $\pi=b-a \rho$. The former equality is, as established in Sect. 3.4, Footnote 9, the relationship between $\rho$ and $\pi$ associated with an utility $L$. The latter equality comes from the optimality conditions. After substitution, the ODE we get for $\rho$ is the one studied in Lemma 2. Now, we resort to Proposition 2, that asserts the existence of $L$ if some assumptions hold. These assumptions are (16)(18), which are automatically fulfilled by $\rho$, as it was explained just after the proof of Lemma 2. Other assumptions are $\theta>0$ and $\Delta>0$ that were also proved in Sect. 3.4. It only remains to impose $\mu_{2}<\mu_{1}$, that is, $a \eta<1$. It is easy to see that this is equivalent to $b<2+a<3$. It is clear that $\phi$ satisfies the necessary optimality conditions by construction. Let us check that it also satisfies the sufficient conditions of Theorem 4. The equilibrium stock evolution is the geometric Brownian motion $d X=$ $(A-N \mu) X d s+\sigma X d \omega$. Now, by Proposition 1 and 2, the utility function satisfies $L^{\prime}(c)=c^{-\mu_{2} / \mu_{1}}\left(\psi_{1} c^{\theta}+\mu_{1}\right)$, with $\mu_{2}<\mu_{1}$ and $\theta>0$. Then, all the hypotheses of Theorem 3 holds by taking a finite-horizon game with limiting function $f(x)=x$ and suitable $S$. It only remains to check the sufficient transversality condition. Computing the conditional expectation of $e^{-r T} V(X(T))$ is easy thanks to (30) and the fact that $X$ is a geometric Brownian motion. In summary, once we replace $\mu_{2} / \mu_{1}=a \eta$ we find two conditions to be fulfilled for the conditional expectation to converge to 0

$$
\begin{aligned}
& (\theta+1-a \eta)\left(A-N \mu+\frac{\sigma^{2}}{2}(\theta-a \eta)\right)<r, \\
& (1-a \eta)\left(A-N \mu-\frac{\sigma^{2}}{2} a \eta\right)<r .
\end{aligned}
$$

The first inequality implies the second one as soon as $a \eta<1$ and $A>N \mu-\frac{\sigma^{2}}{2} a \eta$. The definition of $\eta$ was given above.

\section{Comparative statics and further properties of the MPNE}

The EL Equation, or some variations of it, is useful to reveal important properties of the symmetric MPNE. In this section, we study the following: (i) the turnpike property of 
the MPNE; (ii) the monotonicity of the equilibrium consumption strategy with respect to the resource stock; (iii) the curvature of the equilibrium consumption strategy; (iv) the dependence with respect to the size of the uncertainty; (v) the dependence with respect to variations in the number of players and with respect to the preference rate; and finally, (vi) the issue of extinction.

\subsection{Finite-horizon approximations of the stationary MPNE}

Now we investigate the turnpike properties of the game in the case of CRRA preferences (but not necessarily the linear game, that is, $F$ and $\sigma$ do not need to be linear). We prove next that for any smooth solution $f(x)$ of the stationary EL equation, one can find bequest functions $S$ such that the associated finite-horizon MPNE converges to $f$. This is useful in computing approximated solutions of the stationary EL equation based on well-known methods for the Cauchy problem. In what follows, we study the limit as $T \rightarrow \infty$ of the solution of the EL equation for a finite-horizon $T$, although we do not make the dependence of $\phi(t, x)$ on $T$ explicit so as to shorten notation.

Theorem 7 Consider the game with CRRA instantaneous utility function L and $\alpha<0$. Suppose that $f$ is a solution of the stationary EL Eq. (29). Let $S$ be any bequest function of the finite-horizon game such that $f$ serves as a limiting function. Then, the solution of the finite-horizon EL equation, $\phi(t, x)$, converges to $f(x)$ as $T \rightarrow \infty$.

Proof See "Appendix."

The reverse question, that is, whether the finite-horizon MPNE $\phi(t, x)$ converges to a solution of the stationary EL equation is more difficult. A result in this direction is obtained below for the linear game.

Corollary 2 If $\phi$ is an MPNE for the linear game, then

$$
\lim _{T \rightarrow \infty} \phi(t, x)=-\frac{\beta+\gamma}{\alpha} x,
$$

where $\beta=A+\sigma^{2}(1-\delta) / 2, \gamma=(r-A) / \delta, \alpha=-N+(N-1) / \delta$, that is, it converges to the stationary MPNE of the autonomous game.

Proof Notice that $f(x)=-\frac{\beta+\gamma}{\alpha} x$ is a solution of the stationary EL Eq. (29), thus the result is a consequence of Theorem 7 , simply by taking this limiting function $f$.

\subsection{Monotonicity}

Under mild assumptions, the MPNE is monotonous non-decreasing in the asset stock: the higher the stock of the stochastic productive asset, the higher the consumption is in equilibrium. Once this is shown, we prove that the value function of the players is concave. A direct proof of this fact in a differential game framework (even in the symmetric case) does not seem to follow easily from standard arguments. 
Theorem 8 Assume that the assumptions of Theorem 2 hold and that both $F$ and $S$ are concave. Then the MPNE is non-decreasing in $x$.

Proof See "Appendix."

Corollary 3 Assume that the assumptions of Theorem 8 hold. Then the value function is concave in $x$.

Proof This follows from the shadow price characterization given in the proof of Theorem 3 and from Theorem 8 on the monotonicity of the optimal consumption program, since

$$
V_{x x}(t, x)=\frac{\partial}{\partial x} \lambda(t, x)=\frac{\partial}{\partial x} L^{\prime}(\phi(t, x))=L^{\prime \prime}(\phi(t, x)) \phi_{x}(t, x) \leq 0 .
$$

\subsection{Concavity of the MPNE}

In this section, we study whether the MPNE is concave with respect to the asset level. Carroll and Kimball (1996) proved concavity of the consumption function in a one-player game of finite horizon and discrete time, where uncertainty comes from three sources: labor income, gross interest rate, and discount factor. The family of utility functions considered by those authors were the CRRA class, strictly increasing, concave, and satisfying $L^{\prime \prime \prime} \geq 0$. We limit ourselves to the linear game, but allowing for general functions $S$. Note that Carroll and Kimball (1996) choose $S=0$. Another difference, of course, is that we work in the continuous-time case, with Brownian uncertainty. To prove concavity of the equilibrium consumption function, we will impose that $\varphi$ is concave. This implies a condition both for the instantaneous utility function and the bequest function that is provided in Lemma 3.

Theorem 9 Consider the linear game and suppose also that $\varphi$ is concave. Then the $M P N E$ is concave with respect to $x$.

Proof See "Appendix."

\subsection{Dependence of the MPNE on the uncertainty}

In this section, we study the way the symmetric MPNE depends on the size of uncertainty. We will show that the MPNE is monotonous increasing in $\sigma$, more precisely, we prove that under our assumptions plus a technical hypothesis to allow a change of measure, $\sigma_{1} \leq \sigma_{2}$ implies $\phi^{\sigma_{1}} \leq \phi^{\sigma_{2}}$, where we denote $\phi^{\sigma_{i}}$ the MPNE strategy when the diffusion coefficient $\sigma \in \Sigma$, with $\Sigma$ defined below. To obtain this result is not straightforward, as the equilibrium is driven by a backward SDE, which is coupled with a forward SDE. In our analysis, we will use a variation of the KR rule found in Sect. 5 that represents the equilibrium. Let $\mu=\frac{F}{\sigma}+\frac{\sigma^{\prime}}{2}$ and let 
$\Sigma=\left\{\sigma: \mathbb{R}_{+} \longrightarrow \mathbb{R}_{+}: \mu\right.$ bounded $\}$. For $\sigma \in \Sigma$, let $Y^{\sigma}$ be the process that satisfies (we omit the time argument to simplify notation)

$$
d Y(s)=\left(F(Y)+\left(\sigma \sigma^{\prime}\right)(Y)\right) d s+\sigma(Y) d w(s), \quad Y(t)=x .
$$

Let us define $g_{N}(c)=-N c+(N-1) R(c)$. Since we are supposing in (A3) (a) [see also (12)] that $\alpha^{+}=\rho^{+}(N-1)-N<0$ and $R(c) \leq \rho^{+} c$, then $g_{N}(c) \leq 0$ for all $N \geq 1$ and all $c \geq 0$.

Along $Y^{\sigma}$, the equilibrium satisfies the BSDE

$$
\begin{aligned}
d C(s) & =\left(R(C)\left(F^{\prime}(Y)-r\right)+\frac{1}{2} P(C) C^{2} Z^{2}-\frac{g_{N}(C) Z}{\sigma(Y)}\right) d s+Z d w(s), \\
C(T) & =\varphi(Y(T)),
\end{aligned}
$$

where $Z$ is given by $Z=\sigma\left(Y^{\sigma}(s)\right) \phi_{x}\left(s, Y^{\sigma}(s)\right)$ and $\phi$ is the solution of the EL equation. The proof follows the same lines as the proof of the KR rule in Theorem 5 .

Theorem 10 If both $F$ and $S$ are concave, and $\varphi$ is monotonous non-decreasing, then the MPNE is monotonous non-decreasing with respect to $\sigma \in \Sigma$.

Proof See "Appendix."

In the limit, as the diffusion coefficient tends to zero, and assuming that an MPNE exists of the deterministic game and that convergence holds, we get that the players are more conservative in the deterministic case than when some noise is present. This conclusion holds at every pair of date and stock of the resource, and not only in terms of expected mean growth. Thus, in this model, independently of the sign of the relative prudence index, the players consume at a higher rate as the uncertainty is larger (as measured by the function $\sigma$ ). This result also holds for the one-player case.

\subsection{Variation in the number of players and on the time preference}

Now we will study the dependence of the MPNE with respect to variations in the number of players. The effect of the number of players on the consumption effort depends on the marginal substitution rate $\rho(c)$. When $\rho(c)>1$, consumption increases with $N$, when $\rho(c)<1$ it decreases, and when $\rho(c)=1$ (the logarithm case), it is independent of the number of players. We will use the KR rule to show these facts.

Theorem 11 Suppose that the hypotheses of Theorem 5 hold and let $N_{1} \leq N_{2}$. Then $\phi^{N_{1}} \leq \phi^{N_{2}}$ if $\rho(c) \geq 1$ for any $c \geq 0$, and $\phi^{N_{1}} \geq \phi^{N_{2}}$ if $\rho(c) \leq 1$ for any $c \geq 0$.

Proof See "Appendix."

In the linear game above, the MPNE is non-decreasing with the number of agents if and only if $\delta \leq 1$.

Theorem 12 Suppose that the hypotheses of Theorem 5 hold. If $r_{1} \leq r_{2}$, then $\phi^{r_{1}} \leq$ $\phi^{r_{2}}$.

Proof See "Appendix." 


\subsection{Extinction}

We explore here the question of extinction of the asset in the long run. This has already been investigated in Clemhout and Wan (1985) in the model without uncertainty. These authors proved that with the assumptions $L(0)=0, L^{\prime}(0) \in(0, \infty), L^{\prime \prime}<0, N r<$ $F^{\prime}(0)<\infty$, there exists an $\tilde{x}>0$ such that in equilibrium, any initial stock level $x \in(0, \tilde{x})$ declines to extinction in finite time. This is not true under cartelization. Our main finding is that uncertainty strengthens the competition of the players compared to the deterministic case, and the resource declines to extinction with probability 1 from any initial level of the resource as the time horizon tends to infinity if the number of players is large enough. Mitra and Sorger (2014) study a discrete version of the deterministic model with specific functional forms, and find a necessary and sufficient condition for the resource to not become extinct in equilibrium.

For our purposes, we consider linear $F(x)=A x$ and $\sigma(x)=\sigma x$, but an arbitrary utility function $L$ satisfying our standing assumptions. For a solution $\phi(t, x)$ of the EL Eq. (10) we know from Theorem 2 that

$$
k_{-} f(x) \leq \phi(t, x) \leq k_{+} f(x),
$$

whenever $f$ is a suitable limiting function. Recall the definition of the constants $k_{-}$ and $k_{+}$just above Theorem 3 .

$$
k_{-}=\min \left\{m,-\frac{\beta^{-}}{\alpha^{-} \sup _{(0, \infty)} f^{\prime}}\right\}, \quad k_{+}=\max \left\{M,-\frac{\beta^{+}}{\alpha^{+} \inf _{(0, \infty)} f^{\prime}}\right\},
$$

where $\alpha^{-}, \alpha^{+}$are defined in (12) and $\beta^{-}$and $\beta^{+}$in Assumption (A5) (c). Notice that, given that both $F$ and $\sigma$ are linear, we can also take $f(x)=x$. Hence the MPNE satisfies

$$
k_{-} x \leq \phi(t, x) \leq k_{+} x
$$

for any $t \leq T$, for any $T$ and for any $x \geq 0$. We are free to select a bequest function $S$ such that the associated $\varphi$ satisfies the standing assumption, since we are only interested in the limiting behavior of the solution. The asset follows the SDE

$$
d X(s)=(A X(s)-N \phi(s, X(s))) d s+\sigma X(s) d w(s), \quad X(t)=x .
$$

Let us denote by $X^{\phi}(s ; t, x)$ the unique strong solution. By (37), the drift is bounded by

$$
A X-N c \leq\left(A-N k_{-}\right) X
$$

Consider the SDE

$$
d \bar{X}(s)=\left(A-N k_{-}\right) \bar{X} d s+\sigma \bar{X} d w(s) .
$$


By the classical comparison theorem for SDEs, Ikeda and Watanabe (1977), we have $0 \leq X^{\phi} \leq \bar{X}$ a.s. Hence, if the SDE for $\bar{X}$ is asymptotically stable, the same happens for the SDE for $X$.

Proposition 4 Suppose that

$$
A-N k_{-}<\frac{\sigma^{2}}{2} .
$$

Then the equilibrium $X \equiv 0$ is asymptotically stable in the large, i.e., it is stochastically stable

$$
\lim _{x \rightarrow 0} \mathbf{P}\left\{\sup _{s \geq t}|X(s ; t, x) \geq 0|\right\}=0
$$

and for any initial level of stock $x>0$

$$
\mathbf{P}\left\{\lim _{T \rightarrow \infty} X(T ; t, x)=0\right\}=1 .
$$

Proof The proof is a direct application of Example 2.7 in Mao (1997). For $X=0$ to be asymptotically stable, it suffices that the drift term should be smaller than $\sigma^{2} / 2$.

Inequality (38) depends on several parameters of the game and on the number of players. Since $-\beta^{-} / \alpha^{-}$tends to 0 as $N \rightarrow \infty$ because $\alpha^{-} \rightarrow 0$, we have $k_{-}=$ $-\beta^{-} / \alpha^{-}$for a big enough $N$. Then, for a large number of players $N$

$$
A-N k_{-}=A+\frac{\beta^{-} N}{\left(a^{-}-1\right) N-a^{-}} .
$$

This expression is decreasing with $N$ and in the limit as $N \rightarrow \infty$ inequality (38) is

$$
A+\frac{\beta^{-}}{a^{-}-1}<\frac{\sigma^{2}}{2} .
$$

\section{Conclusions}

The purpose of this paper has been twofold. On the one hand, to provide an easy method to obtain the EL equations of a stochastic differential game where the uncertainty is modeled as a diffusion process and players' decisions cannot influence the size of the uncertainty. On the other hand, to carry out a rigorous study of a symmetric stochastic dynamic game in continuous time where players consume from a productive asset in a noncooperative way. The results obtained prove the usefulness of the EL equations in proving the existence and uniqueness of the MPNE in the game mentioned above, under quite general assumptions. Moreover, it has also been shown how the EL equations are specially suitable to make comparative statics exercises of the equilibrium and to answer important questions of the nature of the dependence of the equilibrium on uncertainty, its curvature, and the issue of extinction. As we 
show, EL equations are equivalent to a stochastic KR rule that is a natural extension of the deterministic one and that shows in a neat way how the uncertainty changes the consumption-saving decisions of the players with respect to the deterministic case. To our knowledge, most of these questions are completely answered for the first time in this paper, thanks to the approach based on the EL equations. Our methods of proof combine methods of partial differential equations to show properties that concern firstor second-order derivatives of the consumption function, as well as the convergence of finite-horizon approximations, together with comparison theorems of FBSDEs for making comparative statics.

With the exception of Sorger (1998), which proves the existence of MPNE in the deterministic infinite-horizon game (but, as explained in the paper, with just the opposite hypothesis that we impose about the elasticity of the marginal utility, and assuming a specific recruitment function), other investigations focus on a particular form of the utility function, the recruitment function, or the diffusion coefficient.

It is by no means trivial to prove the existence of the Nash equilibrium with the property of subgame perfection, even for what we can consider by now is a classical game that has received a lot of attention over the last few decades. As shown in the paper, in our nonexistence result of the MPNE, one cannot freely work with any number of players and any strictly concave and smooth utility function, as it could be that no MPNE exists unless one sets an upper bound in the maximal consumption rate of the players. To address these questions seriously, avoiding heuristic claims that could not be supported on the existence of the object that is analyzed, it seems unavoidable to impose the correct amount of smoothness in the functions defining the game. The following table clarifies the generality with which we attain our results (Table 1). For instance, existence and uniqueness of the MPNE is obtained under our the general hypotheses imposed in the paper (A1-A5), but our conclusions about the dependence on uncertainty require the additional assumption of both the recruitment function and bequest function to be concave. The rest of the table has a similar interpretation.

It is our hope to have had success conveying other researches about the usefulness of the EL equations approach to analyze models from economics, and that they include it as an additional tool for economic analysis. Our aim for the future is to study other

Table 1 List of results obtained and assumptions employed

\begin{tabular}{ll}
\hline Result & $\begin{array}{l}\text { Assumptions on functions } \\
L, S, F, \sigma, \varphi\end{array}$ \\
\hline Existence, uniqueness & General \\
Turnpike & $L$ CRRA \\
Monotonicity & $S$ and $F$ concave \\
Concavity of MPNE & Linear game, $\varphi$ concave and \\
& non-decreasing \\
Dependence on uncertainty & $S$ and $F$ concave \\
Dependence on $N$ and $r$ & General \\
Extinction & $F$ and $\sigma$ linear
\end{tabular}


relevant models with this technique, as well as to extend the method to problems where the players influence the size of the uncertainty through their decisions.

\section{Appendix: Proofs}

In this appendix, we collect the proofs of the results that are not shown in the body text. It also include some auxiliary results that are used in the proofs.

\section{Proof of Theorem 2}

Let $R_{T}=[0, T] \times(0, \infty)$. We first state an auxiliary result.

Existence of solutions to quasilinear parabolic PDEs.

Theorem 13 There exists at least one bounded solution in $R_{T}$ of the Cauchy problem

$$
u_{\tau}-\frac{\partial}{\partial x} a\left(\tau, x, u, u_{x}\right)=b\left(\tau, x, u, u_{x}\right)
$$

with initial condition

$$
u(0, x)=u_{0}(x), \quad x>0 .
$$

if all of the following conditions are satisfied.

C1. $u_{0}$ is of class $C^{4}$ and bounded.

$C 2$. Functions $a$ and $b$ are of class $C^{3}$ and $C^{2}$, respectively.

$C 3$. There are non-negative constants $b_{1}$ and $b_{2}$ such that for all $x$ and $u$

$$
\left(b(\tau, x, u, 0)+\frac{\partial}{\partial x} a(\tau, x, u, 0)\right) u \leq b_{1} u^{2}+b_{2} .
$$

C4. For all $M>0$, there are constants $\mu_{2}(M) \geq \mu_{1}(M)>0$ such that, if $\tau, x$ and $u$ are bounded in modulus by $M$, then for arbitrary $p$

$$
\mu_{1}(M) \leq \frac{\partial}{\partial p} a(\tau, x, u, p) \leq \mu_{2}(M)
$$

and

$$
\left(|a|+\left|\frac{\partial a}{\partial u}\right|\right)(1+|p|)+\left|\frac{\partial a}{\partial x}\right|+|b| \leq \mu_{2}(M)(1+|p|)^{2} .
$$

The problem admits no more than a classical solution in $R_{T}$ that is bounded together with its derivatives of first and second orders if the following additional conditions hold. 
C5. For all $M>0$ there are non-negative constants $v_{1}(M)$ and $\nu_{2}(M)$ such that

$$
\begin{aligned}
& \max _{\substack{(t, x) \in R_{T} \\
|u|,|p| \leq M}}\left|\frac{\partial^{2} a}{\partial p \partial u}, \frac{\partial^{2} a}{\partial p^{2}}, \frac{\partial A}{\partial p}\right| \leq v_{1}(M), \\
& \min _{\substack{(t, x) \in R_{T} \\
|u|,|p| \leq M}} \frac{\partial A}{\partial u} \geq-v_{2}(M),
\end{aligned}
$$

where

$$
A=a-\frac{\partial a}{\partial u} p-\frac{\partial a}{\partial x} .
$$

Proof The result is a consequence of Theorem 8.1 of Ladyzhenskaya et al (1969), the only difference being that we have set the problem in $[0, T] \times(0, \infty)$ instead of $[0, T] \times \mathbb{R}$ and that we require more smoothness. The method of proof of Theorem 8.1 in Ladyzhenskaya et al consists in considering truncated problems on the strip $[0, T] \times[1 / n, n]$ with boundary conditions $u_{n}(0, x)=u_{0}(x)$ for all $x \in[1 / n, n]$ and $^{12} u_{n}(t, 1 / n)=u_{0}(1 / n), u_{n}(t, n)=u_{0}(n)$ for all $t \geq 0$. These solutions converge smoothly to a solution $u$ of the original equation as $n \rightarrow \infty$.

Motivated by the necessity to drop the boundedness of the data defining the game, we will consider the EL Eq. (10) for the function $u=\phi / f$ written in the divergence form

$$
u_{\tau}-\frac{\partial}{\partial x} a\left(x, u_{x}\right)=b\left(x, u, u_{x}\right)
$$

where $\tau=T-t$ and

$$
\begin{aligned}
a(x, p)= & \frac{\sigma(x)^{2}}{2} p \\
b(x, u, p)= & \left(F-N u f+(N-1) R(u f)+\frac{f^{\prime}}{f} \sigma^{2}\right) p \\
& +\left(F-N u f+(N-1) R(u f)+\sigma^{\prime} \sigma\right) u \frac{f^{\prime}}{f} \\
& +\frac{1}{2} \sigma^{2} u \frac{f^{\prime \prime}}{f}-\frac{\sigma^{2}}{2} \frac{P(u f)}{f}\left(p f+u f^{\prime}\right)^{2}+\frac{r-F^{\prime}}{f} R(u f) .
\end{aligned}
$$

The initial condition is

$$
u(0, x)=\varphi_{0}(x)=\frac{\varphi(x)}{f(x)} .
$$

Note that even if the initial condition $\varphi_{0}$ is now bounded by assumption, still we cannot apply the above theorem directly. The difficulties are two: (i) the function $\sigma$ vanishes

\footnotetext{
12 The selection of the boundary conditions at $1 / n$ and $n$ can be done differently, with the only requisite of being compatible with $u_{0}(x)$, that is, conserving continuity and smoothness; in the proof below, we will use a different set of boundary conditions still compatible.
} 
at $x=0$, thus it is not uniformly bounded away from zero; and (ii) the function $P$ is in general not defined at 0 and in fact $\lim _{c \rightarrow 0^{+}} P(c)=\infty$ for problems with CRRA utility, where $P(c)=(1+\delta) / c$. To deal with (i) we consider the PDE (39) on bounded subintervals $I_{n}=[1 / n, n], n=1,2, \ldots$, and then we take a limit as $n \rightarrow \infty$, while for (ii) we will prove that the solutions $u_{n}$ found in the subintervals above remain uniformly bounded away from 0 , in the sense that there exists a lower bound $l_{m}>0$ such that $u_{n} \geq l_{m}$ for all $n \geq m$ for all $x \in I_{m}$. As a byproduct of the proof, we obtain the estimates claimed in the theorem.

- C.1. is fulfilled, since $\varphi_{0}(x)=\varphi(x) / f(x)$ is bounded and smooth on $(0, \infty)$, by assumption.

- C.2. holds, as the function $a$ has the required smoothness. As explained above, we will prove below that $u$ never vanishes on $(0, \infty)$, thus the term $P(u f)$ does not pose any problem at all for the smoothness of function $b$.

- C.3. There are constants $b_{1}$ and $b_{2}$ such that

$$
\begin{aligned}
b(x, u, 0) u= & \left(F-N u f+(N-1) R(u f)+\sigma^{\prime} \sigma\right) u^{2} \frac{f^{\prime}}{f} \\
& +\frac{1}{2} \sigma^{2} u^{2} \frac{f^{\prime \prime}}{f}-\frac{\sigma^{2}}{2} \frac{P(u f)}{f}\left(u f^{\prime}\right)^{2} u+\frac{r-F^{\prime}}{f} R(u f) u \leq b_{1} u^{2}+b_{2}
\end{aligned}
$$

(since $a(x, 0)=0)$. To see this, note that, thanks to our assumptions,

$$
\begin{aligned}
b(x, u, 0) u & \leq \alpha^{+} u^{3} f^{\prime}+\left(\left(F+\sigma^{\prime} \sigma\right) \frac{f^{\prime}}{f}+\frac{1}{2} \sigma^{2} \frac{f^{\prime \prime}}{f}-\frac{\pi^{-}}{2} \sigma^{2}\left(\frac{f^{\prime}}{f}\right)^{2}+\gamma^{+}\right) u^{2} \\
& <\alpha^{+}\left(\min _{x \in(0, \infty)} f^{\prime}\right) u^{3}+\beta^{+} u^{2} \leq \beta^{+} u^{2}
\end{aligned}
$$

since $\alpha^{+}<0$ and the solution $u>0$ (this will be proved below).

- C.4. first part, is also fulfilled, as

$$
\frac{\partial a(x, p)}{\partial p}=\frac{\sigma^{2}(x)}{2}
$$

is positive for $x>0$ and continuous, thus it is bounded away from 0 , as well as bounded above in any compact subset $[1 / M, M]$ of $(0, \infty) .{ }^{13}$ The second part of $C .4$ is a local assumption, that also holds because function $|b|$ is quadratic in $p$, with continuous coefficients, and thus bounded on compact subsets of $(0, \infty)$; the same is true for

$$
\left(|a|+\left|\frac{\partial a}{\partial u}\right|\right)(1+|p|)+\left|\frac{\partial a}{\partial x}\right|=\frac{\sigma^{2}(x)}{2}|p|(1+|p|)+\left|\sigma(x) \sigma^{\prime}(x)\right||p|,
$$

\footnotetext{
13 Note that what we require here is $\mu_{1}(M) \leq \frac{\partial a(x, p)}{\partial p} \leq \mu_{2}(M)$ for $x$ belonging to a compact set of the kind $[1 / M, M]$ and not simply to $x \leq M$. This is a slight variation that is not problematic in this framework.
} 
which is also quadratic in $p$, and since both $\sigma$ and $\sigma^{\prime}$ are continuous and thus they are bounded in compact subsets of $[0, \infty)$.

- C.5. Both $\sigma$ and $\sigma^{\prime}$ are continuous, and therefore bounded over any compact interval of the state space, achieving uniqueness of the bounded solution

We now show that we can construct a solution $u>0$ of the PDE (39) as limit of positive truncated solutions $u_{n}$ in $[0, T] \times[1 / n, n]$ as $n \rightarrow \infty$. Then, $C$.2 holds, as $P$ is smooth in $(0, \infty)$. In fact we prove more than that, as we obtain upper and lower estimates for the solution. The latter will imply in particular that the solution is positive for $x>0$.

Let the PDE (39) with initial condition (40) and boundary conditions at the extreme points of the interval $I_{n}$ given by

$$
\begin{aligned}
u_{n}(\tau, 1 / n) & =\frac{\beta^{+} e^{\tau \beta^{+}} \varphi_{0}(1 / n)}{\alpha^{+} \varphi_{0}(1 / n) f^{\prime}(1 / n)\left(1-e^{\tau \beta^{+}}\right)+\beta^{+}}, \\
u_{n}(\tau, n) & =\frac{\beta^{+} e^{\tau \beta^{+}} \varphi_{0}(n)}{\alpha^{+} \varphi_{0}(n) f^{\prime}(n)\left(1-e^{\tau \beta^{+}}\right)+\beta^{+}} .
\end{aligned}
$$

The reason for this particular selection of the boundary conditions is shown next. Formulating these approximating problems, we have eliminated the degeneration in the truncated equation. It is easy to check that all conditions in Theorem 8.1 of Ladyzhenskaya et al. (1968) are fulfilled in a small neighborhood of $\tau=0$, since $\varphi_{0}(x)$ is bounded away from zero in $I_{n}$ and hence a solution $u_{n}$ of the truncated problem exists which does not vanish. From this, our aim is to extend this truncated solution to all $[0, T]$ and after this, to get the solution $\phi(t, x)$ with initial condition $\phi(0, x)=\varphi(x)$ as the smooth limit of $u_{n}(\tau, x) f(x)$ as $n \rightarrow \infty$. As explained above, this is the procedure used in Ladyzhenskaya et al. (1968). We show next that the local solution can be extended in time and space. To this end, we define

$$
M_{n}(\tau)=\max _{y \in[1 / n, n]} u_{n}(\tau, y)
$$

By Danskin's Theorem, function $M_{n}$ is almost everywhere differentiable, and at points of differentiability, the derivative $\dot{M}_{n}(\tau)=u_{n, \tau}\left(\tau, x_{n}(\tau)\right)$, where $u_{n}\left(\tau, x_{n}(\tau)\right)=$ $M_{n}(\tau)$.

We prove that for any $\tau$

$$
M_{n}(\tau) \leq \frac{\beta^{+} e^{\tau \beta^{+}} M_{n}(0)}{\alpha^{+}\left(\min _{I_{n}} f^{\prime}\right) M_{n}(0)\left(1-e^{\tau \beta^{+}}\right)+\beta^{+}}
$$

where $M_{n}(0)=\sup _{x \in I_{n}} \varphi_{0}(x)$. Suppose, by way of contradiction, that $M_{n}\left(\tau_{0}\right)$ is greater that the right-hand side of (42) for some $\tau_{0}>0$. Let $\bar{\tau}_{0}$ be the inferior of all the $\tau_{0}$ s satisfying this property, and hence, by continuity of $M_{n}, M_{n}\left(\bar{\tau}_{0}\right)$ equals the righthand side of (42). Then $x_{n}(\tau)$ is interior to $I_{n}$ for every $\tau \in\left[\bar{\tau}_{0}, \tau_{0}\right]$, due to the boundary conditions (41). In consequence, $u_{n, x}\left(\tau, x_{n}(\tau)\right)=0$ and $u_{n, x x}\left(\tau, x_{n}(\tau)\right) \leq 0$ for any 
$\tau \in\left[\bar{\tau}_{0}, \tau_{0}\right]$. Hence, $\left.(\partial / \partial x)\left(\sigma^{2} u_{n, x}\right)\right|_{\left(\tau, x_{n}(\tau)\right)} \leq 0$. This information, used in the Eq. (39) for $v_{n}$, provides the following chain of inequalities

$$
\begin{aligned}
\dot{M}_{n}(\tau) \leq & b\left(x_{n}(\tau), M_{n}(\tau), 0\right) \\
= & \left(F-N f M_{n}(\tau)+(N-1) R\left(f M_{n}(\tau)\right)+\sigma^{\prime} \sigma\right) M_{n}(\tau) \frac{f^{\prime}}{f} \\
& +\frac{1}{2} \sigma^{2} M_{n}(\tau) \frac{f^{\prime \prime}}{f}-\frac{1}{2} \sigma^{2} \frac{P\left(f M_{n}(\tau)\right)}{f}\left(M_{n}(\tau) f^{\prime}\right)^{2}+\frac{r-F^{\prime}}{f} R\left(f M_{n}(\tau)\right) \\
\leq & \alpha^{+} M_{n}^{2}(\tau) f^{\prime}+\left(\left(F+\sigma^{\prime} \sigma\right) \frac{f^{\prime}}{f}+\frac{1}{2} \sigma^{2} \frac{f^{\prime \prime}}{f}-\frac{\pi^{-}}{2} \sigma^{2}\left(\frac{f^{\prime}}{f}\right)^{2}+\gamma^{+}\right) M_{n}(\tau) \\
< & \alpha^{+}\left(\min _{I_{n}} f^{\prime}\right) M_{n}^{2}(\tau)+\beta^{+} M_{n}(\tau), \text { a.e. } \tau .
\end{aligned}
$$

We have used the definitions of $\alpha^{+}$and $\beta^{+}$done in Assumption (A3) and (A5), respectively, and the fact that $\alpha^{+}<0$. From this differential inequality of Ricatti we get the estimate

$$
M_{n}(\tau) \leq \frac{\beta^{+} e^{\left(\tau-\bar{\tau}_{0}\right) \beta^{+}} M_{n}\left(\bar{\tau}_{0}\right)}{\alpha^{+}\left(\min _{I_{n}} f^{\prime}\right) M_{n}\left(\bar{\tau}_{0}\right)\left(1-e^{\left(\tau-\bar{\tau}_{0}\right) \beta^{+}}\right)+\beta^{+}} .
$$

Once the expression for $M_{n}\left(\bar{\tau}_{0}\right)=\frac{\beta^{+} e^{\bar{\tau}} \beta^{+} M_{n}(0)}{\alpha^{+}\left(\min _{I_{n}} f^{\prime}\right) M_{n}(0)\left(1-e^{\bar{\tau}} \beta^{+}\right)+\beta^{+}}$, which holds by our contradiction argument is substituted into (A.1) inequality (42) easily follows.

Following the same technique, we get the lower estimate. Consider the solution $v_{n}$ of the PDE with the boundary conditions

$$
\begin{aligned}
v_{n}(\tau, 1 / n) & =\frac{\beta^{-} e^{\tau \beta^{-}} \varphi_{0}(1 / n)}{\alpha^{-} \varphi_{0}(1 / n) f^{\prime}(1 / n)\left(1-e^{\tau \beta^{-}}\right)+\beta^{-}}, \\
v_{n}(\tau, n) & =\frac{\beta^{-} e^{\tau \beta^{-}} \varphi_{0}(n)}{\alpha^{-} \varphi_{0}(n) f^{\prime}(n)\left(1-e^{\tau \beta^{-}}\right)+\beta^{-}} .
\end{aligned}
$$

and

$$
m_{n}(\tau)=\min _{y \in[1 / n, n]} v_{n}(\tau, y)
$$

with the minimum attained at some $y_{n}(\tau)$. Similar arguments as done above for the maximum lead to the estimate

$$
m_{n}(\tau) \geq \frac{\beta^{-} e^{\tau \beta^{-}} m_{n}(0)}{\alpha^{-} m_{n}(0)\left(\max _{I_{n}} f^{\prime}\right)\left(1-e^{\tau \beta^{-}}\right)+\beta^{-}} .
$$


as follows. By way of contradiction one finds

$$
\dot{m}_{n}(\tau) \geq g\left(y_{n}(\tau), m_{n}(\tau), 0\right) \geq \alpha^{-}\left(\max _{I_{n}} f^{\prime}\right) m_{n}^{2}(\tau)+\beta^{-} m_{n}(\tau), \quad \text { a.e. } \tau \text {. }
$$

Reasoning much as for the case of $M_{n}$, one gets the estimate (44) easily. Thus, we have shown that the local solution is strictly uniformly bounded away from zero in the intervals $I_{n}$ and that it is also bounded above. Since this fact is independent of $\tau$, as well as the upper bound obtained above, the solution can be extended up to the whole $[0, T]$, for any $T$.

Hence, since $v_{n} \rightarrow v$, we have that $v$ is bounded away from zero, and hence $v$ is a solution of the Cauchy problem, since all the conditions of the Theorem of Ladyzhenskaya et al. (1968) are fulfilled. Taking limits as $n \rightarrow \infty$ one has

$$
\frac{\phi(t, x)}{f(x)}=u(T-t, x) \geq m(T-t) \geq \frac{\beta^{-} e^{(T-t) \beta^{-}} m(0)}{\alpha^{-} m(0)\left(\sup _{(0, \infty)} f^{\prime}\right)\left(1-e^{(T-t) \beta^{-}}\right)+\beta^{-}} .
$$

By the above estimates the limit $\phi(t, x)=u(T-t, x) f(x)$ is a solution of the Cauchy problem (10) satisfying

$$
\frac{\phi(t, x)}{f(x)}=v(T-t, x) \leq M(T-t) \leq \frac{\beta^{+} e^{(T-t) \beta^{+}} M}{\alpha^{+}\left(\inf _{(0, \infty)} f^{\prime}\right) M\left(1-e^{(T-t) \beta^{+}}\right)+\beta^{+}},
$$

since $M_{n}(0) \rightarrow M$ as $n \rightarrow \infty$, and $\inf _{(0, \infty)} f^{\prime} \leq \min _{I_{n}} f^{\prime}$.

\section{Proof of Theorem 7}

With CRRA preferences, $\rho^{+}=\rho^{-}=\rho=1 / \delta, \alpha^{+}=\alpha^{-}=\alpha$ and $\pi^{+}=\pi^{-}=$ $b=1+\delta$. See the definition of these constants in Sect. 3, Assumption (A3) and in (12). See also the computations done after Definition 4. We will follow the proof of Theorem 2, using the same notation. Retaking inequality (43) in the aforementioned proof, in the case of CRRA preferences we have

$$
\begin{aligned}
\dot{M}_{n}(\tau) \leq & b\left(x_{n}(\tau), M_{n}(\tau), 0\right) \\
= & \left(F-N f M_{n}(\tau)+(N-1) a f M_{n}(\tau)+\sigma^{\prime} \sigma\right) M_{n}(\tau) \frac{f^{\prime}}{f} \\
& +\frac{1}{2} \sigma^{2} M_{n}(\tau) \frac{f^{\prime \prime}}{f}-\frac{1}{2} \sigma^{2} \frac{b}{f^{2} M_{n}(\tau)}\left(M_{n}(\tau) f^{\prime}\right)^{2}+\frac{r-F^{\prime}}{f} M_{n}(\tau) a \\
\leq & \alpha f^{\prime} M_{n}^{2}(\tau)+\left(\left(F+\sigma^{\prime} \sigma\right) f^{\prime}+\frac{1}{2} \sigma^{2} f^{\prime \prime}-\frac{1}{2} \sigma^{2} \frac{b}{f} f^{\prime 2}+a\left(r-F^{\prime}\right) f\right) \frac{M_{n}(\tau)}{f}
\end{aligned}
$$

Since $f$ is solution of the stationary EL Eq. (10) with CRRA preferences, then

$$
\left(F+\sigma^{\prime} \sigma\right) f^{\prime}+\frac{1}{2} \sigma^{2} f^{\prime \prime}-\frac{1}{2} \sigma^{2} \frac{b}{f} f^{\prime 2}+\left(r-F^{\prime}\right) a f=-\alpha f f^{\prime} .
$$


Plugging this into the inequality above it simplifies to

$$
\dot{M}_{n}(\tau) \leq \alpha f^{\prime} M_{n}^{2}(\tau)-\alpha f^{\prime} M_{n}(\tau) \leq \alpha\left(\inf _{I_{n}} f^{\prime}\right) M_{n}^{2}(\tau)-\alpha\left(\sup _{I_{n}} f^{\prime}\right) M_{n}(\tau)
$$

Hence

$$
M_{n}(\tau) \leq \frac{M_{n}(0) e^{-\tau \alpha\left(\sup _{I_{n}} f^{\prime}\right)}}{-M_{n}(0)\left(1-e^{-\tau \alpha\left(\sup _{I_{n}} f^{\prime}\right)}\right)+1} .
$$

A similar computation for the minimum shows

$$
m_{n}(\tau) \geq \frac{m_{n}(0) e^{-\tau \alpha\left(\inf _{I_{n}} f^{\prime}\right)}}{-m_{n}(0)\left(1-e^{-\tau \alpha\left(\inf _{I_{n}} f^{\prime}\right)}\right)+1} .
$$

Notice that in the former case $f^{\prime}$ is evaluated at the point where $u_{n}$ attains a maximum in $I_{n}=[1 / n, n]$, say $x_{n}$, whereas in the latter case it is at the point where $u_{n}$ attains a minimum, say $y_{n}$. Thus,

$$
\frac{m_{n}(0) e^{-\tau \alpha\left(\inf _{I_{n}} f^{\prime}\right)}}{-m_{n}(0)\left(1-e^{-\tau \alpha\left(\inf _{I_{n}} f^{\prime}\right)}\right)+1} \leq u_{n}(t, x) \leq \frac{M_{n}(0) e^{-\tau \alpha\left(\sup _{I_{n}} f^{\prime}\right)}}{-M_{n}(0)\left(1-e^{-\tau \alpha\left(\sup _{I_{n}} f^{\prime}\right)}\right)+1} .
$$

Taking the limit as $n$ tends to $\infty$ and since $u_{n}(t, x) \rightarrow \phi(t, x) / f(x)$ as $n \rightarrow \infty$, we find

$$
\frac{m e^{-\tau \alpha\left(\inf _{I_{n}} f^{\prime}\right)}}{-m\left(1-e^{-\tau \alpha\left(\inf _{I_{n}} f^{\prime}\right)}\right)+1} \leq \frac{\phi(\tau, x)}{f(x)} \leq \frac{M e^{-\tau \alpha\left(\sup _{I_{n}} f^{\prime}\right)}}{-M\left(1-e^{-\tau \alpha\left(\sup _{I_{n}} f^{\prime}\right)}\right)+1} .
$$

As $T \rightarrow \infty, \tau \rightarrow \infty$ and one finally find that $\phi(t, x)$ converges to $f(x)$.

\section{Proof of Theorem 8}

We follow the same scheme of proof as in Theorems 2 and 7. Notice that the hypotheses of the theorem imply $\varphi^{\prime}(x)=S^{\prime \prime}(x) / L^{\prime \prime}\left(S^{\prime}(x)\right) \geq 0$. Now, derive the EL Eq. (10) with respect to $x$ and let $v=\phi_{x}$. Then $w$ solves the Cauchy problem

$$
\begin{aligned}
v_{\tau}-\frac{1}{2} \frac{\partial}{\partial x}\left(\sigma(x)^{2} v_{x}\right) & =g_{x}(x, \phi, v)+v g_{c}(x, \phi, v)+v_{x} g_{v}(x, \phi, v) \\
+v \sigma^{\prime}(x)^{2}+v \sigma(x) \sigma^{\prime \prime}(x)+v_{x} \sigma(x) \sigma^{\prime}(x) . & \\
v(0, x) & =\varphi^{\prime}(x) \geq 0 .
\end{aligned}
$$

Function $g$ is

$$
g(x, c, v)=(F(x)-N c+(N-1) R(c)) v-\frac{1}{2} \sigma(x)^{2} P(c) v^{2}+\left(r-F^{\prime}(x)\right) R(c) .
$$


We will follow a similar strategy of proof as in Theorem 2, considering truncated intervals for the variable $x, I_{n}=[1 / n, n]$ and the solution $v_{n}(\tau, x)$ in $[0, T] \times I_{n}$ satisfying the boundary conditions

$$
v_{n}(\tau, 1 / n)=\varphi^{\prime}(1 / n)>0, \quad v_{n}(\tau, n)=\varphi^{\prime}(n)>0
$$

Let $v_{n}(\tau)=\min _{x \in I_{n}} v_{n}(\tau, x)$. A reasoning by contradiction, assuming the existence of $\tau_{0}$ satisfying $v_{n}\left(\tau_{0}\right)<v_{n}(0)$, will lead to a contradiction as follows. Let $\bar{\tau}_{0}$ be the inferior of all the $\tau$ s satisfying this property; by continuity of $v_{n}, v_{n}\left(\bar{\tau}_{0}\right)=v_{n}(0)$. Then, we obtain the inequality

$$
\dot{v}_{n} \geq g_{x}\left(x, \phi, v_{n}\right)+v_{n}\left(g_{c}\left(x, \phi_{n}, v_{n}\right)+\sigma^{\prime}(x)^{2}+\sigma(x) \sigma^{\prime \prime}(x)\right) \text {. }
$$

We have used the same arguments as those used in the proof of Theorem 2, hence we do not repeat it here. The notation $\phi_{n}$ is used for the solution of the EL Eq. (10) in $[0, T] \times I_{n}$. The term $\phi_{n}(\tau, \zeta(\tau))$, where $\zeta(\tau)$ minimizes $v_{n}$ over $I_{n}$, does not pose any problem at all. Given that $g_{x}(x, c, v)=v\left(F^{\prime}(x)-v \sigma(x) \sigma^{\prime}(x) P(c)\right)-F^{\prime \prime}(x) R(c)$, we have

$$
\begin{aligned}
\dot{v}_{n} \geq & -F^{\prime \prime}(x) R\left(\phi_{n}\right) \\
& +v_{n}\left(F^{\prime}(x)-v_{n} \sigma(x) \sigma^{\prime}(x) P\left(\phi_{n}\right)+g_{c}\left(x, \phi_{n}, v_{n}\right)+\sigma^{\prime}(x)^{2}+\sigma(x) \sigma^{\prime \prime}(x)\right),
\end{aligned}
$$

with $v_{n}(\bar{\tau})=0$. Since $F^{\prime \prime}$ is concave and $R$ is non-negative, $-F^{\prime \prime}(x) R(c) \geq 0$, thus

$$
v_{n}(\tau) \geq v_{n}\left(\bar{\tau}_{0}\right) e^{\int_{\bar{\tau}_{0}}^{\tau}\{\cdots\} d \tau}=0 \quad \forall \tau \in\left[\bar{\tau}_{0}, \tau_{0}\right]
$$

in contradiction with $v_{n}\left(\tau_{0}\right)<0$. Hence $0 \leq v_{n}(\tau) \leq v_{n}(\tau, x)$ for all $\tau, x \in I_{n}$, and then the limit function $\phi_{x}(\tau, x) \geq 0$.

\section{Proof of Theorem 9}

We first establish a lemma about the concavity of the consumption rate at the final time $T$. It is established for general $L$, not only in the class CRRA. In the lemma, $\rho_{\{\}}$ stands for the elasticity of the marginal utility and $\pi_{\{\}}$for the relative prudence index of a given utility function. For the linear game, the lemma implies that $\varphi$ is concave if and only if the bequest function $S$ satisfies

$$
\frac{S^{\prime}(x) S^{\prime \prime \prime}(x)}{S^{\prime \prime 2}(x)} \geq 1+\frac{1}{\delta} .
$$

Lemma $3 \varphi^{\prime \prime} \leq 0$ if and only if for all $x>0$

$$
\rho_{S}(x) \pi_{S}(x) \geq \rho_{L}(\varphi(x)) \pi_{L}(\varphi(x)) .
$$


Proof Deriving twice in $L^{\prime}(\varphi(x))=S^{\prime}(x)$ we get

$$
\begin{aligned}
& L^{\prime \prime}(\varphi(x)) \varphi^{\prime}(x)=S^{\prime \prime}(x), \\
& L^{\prime \prime \prime}(\varphi(x)) \varphi^{\prime}(x)^{2}+L^{\prime \prime}(\varphi(x)) \varphi^{\prime \prime}(x)=S^{\prime \prime \prime}(x) .
\end{aligned}
$$

Solving for $\varphi^{\prime \prime}(x)$ and imposing $\varphi^{\prime \prime}(x) \leq 0$ we obtain the inequality (we eliminate arguments)

$$
\frac{S^{\prime \prime \prime}}{S^{\prime \prime 2}} \geq \frac{L^{\prime \prime \prime}}{L^{\prime \prime 2}}
$$

or equivalently, multiplying both sides of the inequality by $S^{\prime}>0$

$$
\left(\frac{-x S^{\prime \prime \prime}}{S^{\prime \prime}}\right)\left(\frac{-S^{\prime}}{x S^{\prime \prime}}\right) \geq\left(\frac{-\varphi L^{\prime \prime \prime}}{L^{\prime \prime}}\right)\left(\frac{-S^{\prime}}{\varphi L^{\prime \prime}}\right) .
$$

Noting that $S^{\prime}=L^{\prime}(\varphi)$, and plugging this equality into the right-hand side of the inequality above, we obtain the claim of the lemma.

We now proceed with the proof of Theorem 9 with the same techniques as those used in the proofs of Theorems 2 and 8, deriving twice in the EL Eq. (10) to find a PDE for $\phi_{x x}$. We refer the reader to the proofs of those theorems for filling in the missing details. Deriving twice in (10) we get

$$
\left(\phi_{x x}\right)_{\tau}-\frac{\partial}{\partial x}\left(\frac{\sigma^{2} x^{2}}{2} \phi_{x x x}\right)=j\left(x, \phi, \phi_{x}, \phi_{x x}, \phi_{x x x}\right)
$$

where the function $j$ is defined as

$$
\begin{aligned}
j(x, c, v, w, z)= & w\left(3 \sigma^{2}+2 F^{\prime}(x)+3 \alpha v+\frac{r-F^{\prime}(x)}{\delta}-4 \sigma^{2} x \frac{1+\delta}{c} v\right. \\
& \left.+\frac{5}{2} \sigma^{2} x^{2} \frac{1+\delta}{c^{2}} v^{2}-\sigma^{2} x^{2} \frac{1+\delta}{c} w\right) \\
& -\sigma^{2} v^{2} \frac{1+\delta}{c}\left(1-\frac{x v}{c}\right)^{2} \\
& -F^{\prime \prime \prime}(x) \frac{c}{\delta}+F^{\prime \prime}(x)\left(1-\frac{2}{\delta}\right) v \\
& +\left(2 \sigma^{2} x+F(x)+g_{N}(c)-\sigma^{2} x^{2} \frac{1+\delta}{c} v\right) z .
\end{aligned}
$$

Recall that in the CRRA case, $\alpha=-N+(N-1) / \delta$. Following the same method of proof as in the above referenced theorems, and defining $w_{n}$ as the solution of the PDE above in the interval $I_{n}$, we get

$$
w_{n, \tau}-\frac{\partial}{\partial x}\left(\frac{\sigma^{2} x^{2}}{2} w_{n, x}\right)=j\left(x, \phi_{n}, \phi_{n, x}, w_{n}, w_{n, x}\right) .
$$


Here, $\phi_{n}$ denotes the restriction of $\phi$ to $[0, T] \times I_{n}$. Let $\omega_{n}(\tau)=\max _{I_{n}} w_{n}(\tau, x)$. Reasoning by contradiction supposing that $\omega_{n}(\tau)>0$ at some $\bar{\tau}>0$, one has that $w_{n, x}(\bar{\tau})=0$ and $\frac{\partial}{\partial x}\left(\frac{\sigma^{2} x^{2}}{2} w_{n, x}\right) \leq 0$, thus plugging this into Eq. (45) we get

$\dot{\omega}_{n} \leq \omega_{n}\{\cdots\}-\sigma^{2} \phi_{n, x}^{2} \frac{1+\delta}{\phi_{n}}\left(1-\frac{x \phi_{n, x}}{\phi_{n}}\right)^{2}-F^{\prime \prime \prime}(x) \frac{\phi_{n}}{\delta}+F^{\prime \prime}(x)\left(1-\frac{2}{\delta}\right) \phi_{n, x}$.

In the linear game $F^{\prime \prime}=F^{\prime \prime \prime}=0$, hence the second summand in the above expression is non-positive. Given that $\omega_{n}(0)=\sup _{[0, \infty)} \varphi^{\prime \prime}(x) \leq 0$ is also non-positive, we arrive to a contradiction, because it is never possible to have $\omega_{n}(\bar{\tau})>0$ from the above estimate for $\dot{\omega}_{n}$.

In the following proofs, recall that $g_{N}(c)=-N c+(N-1) R(c)$.

\section{Proof of Theorem 10}

Let us show that if $\sigma_{i} \in \Sigma, i=1,2$ and $\sigma_{1} \leq \sigma_{2}$, then $Y^{\sigma_{1}} \leq Y^{\sigma_{2}}$ a.s. Clearly, for $\sigma \in \Sigma, \mu(Y(s))$ satisfies the Novikov condition and then

$$
M(s)=\exp \left\{\int_{t}^{s} \mu(Y(a)) d(a)-\frac{1}{2} \int_{t}^{s} \mu^{2}(Y(a)) d a\right\}, \quad s \in[t, T]
$$

is a $\mathbf{P}$-martingale, where $\mathbf{P}$ is the objective probability measure. Define now the probability measure $\widetilde{\mathbf{P}}$ by

$$
\frac{d \widetilde{\mathbf{P}}}{d \mathbf{P}}=M(T)
$$

It is known that $\widetilde{\mathbf{P}}$ is absolutely continuous with respect to $\mathbf{P}$. By Girsanov's Theorem, $\widetilde{\omega}(s)=\omega(s)-\int_{t}^{s} \mu(Y(a)) d(a)$ is a $\widetilde{\mathbf{P}}$-Brownian motion and, in the new measure, $Y$ satisfies

$$
d Y(s)=\frac{1}{2} \sigma(Y(s)) \sigma^{\prime}(Y(s)) d s+\sigma(Y(s)) d w(s)
$$

Now, given that $0<\sigma_{1} \leq \sigma_{2}$, it holds that $\int_{x}^{y} \frac{d z}{\sigma_{1}(z)} \geq \int_{x}^{y} \frac{d z}{\sigma_{2}(z)}$ for all $y \geq x$, which is the sufficient condition of Example 2 of Zhiyuan (1984) assuring $Y^{\sigma_{1}} \leq Y^{\sigma_{2}}$ for every $s \in[t, T], \widetilde{\mathbf{P}}$-a.s., whence $\mathbf{P}$-a.s. Then $\varphi\left(Y^{\sigma_{1}}(T)\right) \leq \varphi\left(Y^{\sigma_{2}}(T)\right) \mathbf{P}$-a.s., since $\varphi$ is non-decreasing by hypothesis.

Now, let us define $f^{i}(\omega, s, C, Z)$ as the drift term in the backward SDE for $C$ in (36) when the forward process is $Y^{\sigma_{i}}(s), i=1,2$, that is

$$
f^{i}(\omega, s, C, Z)=C\left(\rho(C)\left(F^{\prime}\left(Y^{\sigma_{i}}\right)-r\right)+\frac{1}{2} \pi(C) Z^{2}-\frac{g_{N}(C) Z}{\sigma\left(Y^{\sigma_{i}}\right)}\right),
$$


as well as $\varphi^{i}(T)=\varphi\left(Y^{\sigma_{i}}(T)\right)$. In the above, $\omega$ denotes an element of the sample space $\Omega$ where the probability $\mathbf{P}$ is defined, in order to stress the dependence of the drift term with respect to the stochastic process $Y^{\sigma_{i}}$. Let also $\left(C^{1}, Z^{1}\right),\left(C^{2}, Z^{2}\right)$ be solutions of the BSDE (36) when $\sigma=\sigma_{i}, i=1,2$. We can write (36) in integral form

$$
C^{i}(s)=\varphi^{i}-\int_{s}^{T} f^{i}(\omega, v, C(v), Z(v)) d v-\int_{s}^{T} Z^{i}(v) d w(v) .
$$

Let us check that $-f^{1}\left(\omega, s, C^{2}, Z^{2}\right) \leq-f^{2}\left(\omega, s, C^{1}, Z^{1}\right)$. Note that there are two terms that depend on $i$ in the definition of $f^{i}$ : one is $-\frac{g_{N}(C) Z}{\sigma\left(Y^{\sigma_{i}}\right)}$; we have already proved that $\sigma\left(Y^{\sigma_{1}}\right) \leq \sigma\left(Y^{\sigma_{2}}\right)$. Since $\sigma$ is increasing, that $Z \geq 0$ for all $s \mathbf{P}$-a.s., and that $g_{N}(C) \leq 0$ (see Sect. 5), then $-\frac{g_{N}(C)}{\sigma\left(Y^{\sigma_{2}}\right)} \leq-\frac{g_{N}(C)}{\sigma\left(Y^{\sigma_{1}}\right)}$. The other term is $F^{\prime}\left(Y^{\sigma_{i}}\right)$; since $F$ is concave, $F^{\prime}$ is non-increasing, thus $-F^{\prime}\left(Y^{\sigma_{1}}\right) \leq-F^{\prime}\left(Y^{\sigma_{2}}\right)$. Hence, $-f^{1}\left(\omega, s, C^{2}, Z^{2}\right) \leq-f^{2}\left(\omega, s, C^{1}, Z^{1}\right)$, as claimed. Now, the Comparison Theorem 2.2 in Karoui et al. (1997) ensures that $C^{1}(s) \leq C^{2}(s)$ for all $s$, P-a.s. Since the process $C^{i}$ is deterministic at $(t, x)$ and $C^{i}(t)=\phi^{\sigma^{i}}(t, x)$, we have that $\phi^{\sigma_{1}}(t, x) \leq \phi^{\sigma_{2}}(t, x)$ and the proof is finished.

\section{Proof of Theorem 11}

To show the result, we will use a representation of the MPNE by means of an FBSDE, alternative to the KR rule introduced in Sect. 5, more amenable for our purposes. Let the process $Y$ that satisfies

$$
d Y=\left(F(Y)+\left(\sigma \sigma^{\prime}\right)(Y)\right) d s+\sigma(Y) d \omega, \quad Y(t)=x,
$$

where we have omitted the argument $s$ to shorten notation. Then, for $C(s)=$ $\phi(s, Y(s))$ and the square-integrable adapted process $\left(Z=\sigma(Y(s)) \phi_{x}(s, Y(s))\right)$, where $\phi$ is a solution of the EL Eq. (10) we have, as in Sect. 6.4

$$
\begin{aligned}
d C(s)= & \left(R(C(s))\left(F^{\prime}(Y(s))-r\right)-\frac{1}{2} P(C(s)) Z^{2}(s)+\frac{g_{N}(C(s))}{\sigma(Y(s))} Z(s)\right) d s \\
& +Z(s) d w(s),
\end{aligned}
$$

and $C(T)=\varphi(Y(T))$. Note that the process $Z$ does not depend on the number of players, $N$. Let

$$
f^{N}(\omega, s, C, Z)=R(C)\left(F^{\prime}(Y(s))-r\right)-\frac{1}{2} P(C) Z^{2}+\frac{g_{N}(C)}{\sigma(Y(s))} Z
$$

be the drift term of the SDE of $C$ when the number of players is $N$ and let $\left(C^{N}, Z^{N}\right)$ be the corresponding solution. We have included $\omega \in \Omega$ into the notation to stress the dependence with respect to the process $Y$. We know that $g_{N}$ is negative thanks to Assumption (A3) (a) (see also (12)). On the other hand, it is easy to see that $g_{N}$, as a function of $N$, is monotonous increasing if $\rho(c)>1$ and decreasing if $\rho(c)<1$. Let 
us suppose first that $\rho(c)>1$. Given that $Z \geq 0$ a.s. by Theorem 5 , then $\frac{g_{N_{1}}(C)}{\sigma} Z \leq$ $\frac{g_{N_{2}}(C)}{\sigma} Z$ a.s. if $N_{1} \leq N_{2}$. Hence $-f^{N_{1}}\left(\omega, s, C^{N_{2}}, Z^{N_{2}}\right) \leq-f^{N_{2}}\left(\omega, s, C^{N_{2}}, \xi^{N_{2}}\right)$, as well as $C^{N_{1}}(T)=C^{N_{2}}(T)$. According to the Comparison Theorem 2.2 of Karoui et al. (1997), $C^{N_{1}}(s) \leq C^{N_{2}}(s)$ for all $t \leq s \leq T$, a.s. The case $\rho(c)<1$ is analyzed analogously. Since $\phi^{N_{i}}(t, x)=\bar{C}^{N_{i}}(t)$ is deterministic, the theorem is proved.

\section{Proof of Theorem 12}

We follow the same steps as in the proof of Theorem 11, using the same FBSDE representation. The process $Y$ is independent of the preference rate and the drift term of the SDE of $C$ in (A.6), $f^{r}$, is decreasing in the preference rate. Hence $r_{1} \leq r_{2}$ implies $-f^{r_{1}} \leq-f^{r_{2}}$ and, by the Comparison Theorem 2.2 of Karoui et al. (1997), we have the result.

\section{References}

Amir, R.: Continuous stochastic games of capital accumulation with convex transitions. Games Econ. Behav. 15, 111-131 (1996a)

Amir, R.: Strategic intergenerational bequests with stochastic convex production. Econ. Theory 8, 367-376 (1996b)

Amir, R.: A new look at optimal growth under uncertainty. J. Econ. Dyn. Control 22, 67-86 (1997)

Balbus, Ł., Reffett, K., Wózny, Ł.: A constructive study of Markov equilibria in stochastic games with strategic complementarities. J. Econ. Theory 150, 815-840 (2014)

Başar, T., Olsder, G.J.: Dynamic Non-Cooperative Game Theory. Society for Industrial and Applied Mathematics, Philadelphia (1999)

Benhabib, J., Radner, R.: The joint exploitation of a productive asset: a game-theoretic approach. Econ. Theory 2, 155-190 (1992)

Bismut, J.M.: Conjugate convex functions in optimal stochastic control. J. Math. Anal. Appl. 44, 384-404 (1973)

Bourguignon, F.: A particular class of continuous-time stochastic growth models. J. Econ. Theory 9, 141158 (1974)

Carroll, C.D., Kimball, M.S.: On the concavity of the consumption function. Econometrica 64, 981-992 (1996)

Case, J.: A dynamic version of Cournot's problem. In: Roxin, O., Liu, P.T., Sternbergm, R.L. (eds.) Differential Games and Control Theory, Lecture Notes In Pure And Applied Mathematics vol 10, pp. 161-177. Dekker, New York (1974)

Chang, F.R.: The inverse optimal problem: a dynamic programming approach. Econometrica 56, 147-172 (1988)

Conrad, J.M.: Differential games in a stochastic fishery. Unpublished manuscript (2012)

Clemhout, S., Wan Jr, H.Y.: Cartelization conserves endangered species? In: Feichtinger, G. (ed.) Optimal Control Theory and Economic Analysis 2, pp. 549-568. North-Holland, Amsterdam (1985)

Danskin, J.M.: The theory of max-min, with applications. SIAM J. Appl. Math. 14, 641-664 (1966)

Dockner, E., Jorgensen, S., Van Long, N., Sorger, G.: Differential Games in Economics and Management Science. Cambridge University Press, Cambridge (2000)

Dockner, E., Sorger, G.: Existence and properties of equilibria for a dynamic game on productive assets. J. Econ. Theory 71, 209-227 (1996)

Dockner, D., Wagener, F.: Markov perfect Nash equilibria in models with a single capital stock. Econ. Theory 56, 585-625 (2014)

Dutta, P.K., Sundaram, R.K.: How different can strategic models be? J. Econ. Theory 60, 42-61 (1993a)

Dutta, P.K., Sundaram, R.K.: The tragedy of the commons? Econ. Theory 3, 413-426 (1993b) 
El Karoui, N., Peng, S., Quenez, M.C.: Backward stochastic differential equations in finance. Math. Finance 7, 1-71 (1997)

Fleming, W.H., Soner, H.M.: Controlled Markov Processes and Viscosity Solutions. Springer, New York (2006)

Foldes, L.: The optimal consumption function in a Brownian model of accumulation. Part A: the consumption function as solution of a boundary value problem. J. Econ. Dyn. Control 25, 1951-1971 (2001)

Ikeda, N., Watanabe, S.: A comparison theorem for solutions of stochastic differential equations and its applications. Osaka J. Math. 14, 619-633 (1977)

Josa-Fombellida, R., Rincón-Zapatero, J.P.: New approach to stochastic optimal control. J. Optim. Theory Appl. 135, 163-177 (2007)

Josa-Fombellida, R., Rincón-Zapatero, J.P.: On a PDE arising in one-dimensional stochastic control problems. J. Optim. Theory Appl. 146, 1-26 (2010)

Kamihigashi, T.: Almost sure convergence to zero in stochastic growth models. Econ. Theory 29, 231-237 (2006)

Kimball, M.S.: Precautionary saving in the small and in the large. Econometrica 58, 53-73 (1990)

Ladyzhenskaya, O.A., Solonnikov, V.A., Ural'ceva, N.N.: Linear and Quasi-Linear Equations of Parabolic Type. Translations of Mathematical Monographs, vol. 23. American Mathematical Society, Providence (1968)

Lancaster, K.: The dynamic inefficiency of capitalism. J. Polit. Econ. 87, 1092-1109 (1973)

Leland, H.E.: Saving and uncertainty: the precautionary demand for saving. Q. J. Econ. 82, 465-473 (1968)

Levhari, D., Mirman, L.J.: The great fish war: an example using a dynamic Cournot-Nash solution. Bell J. Econ. 1, 322-334 (1980)

Ma, J., Yong, J.: Forward-Backward Stochastic Differential Equations and Their Applications. Lecture Notes in Mathematics 1702. Springer, Berlin (1999)

Mao, X.: Stochastic Differential Equations and Their Applications. Horwood Publishing, Chichester (1997)

Martín-Herrán, G., Rincón-Zapatero, J.P.: Efficient Markov perfect Nash equilibria: theory and application to dynamic fishery games. J. Econ. Dyn. Control 29, 1073-1096 (2005)

Melhmann, A.: Applied Differential Games. Plenum Press, New York (1988)

Merton, R.C.: An assymptotic theory of growth under uncertainty. Rev. Econ. Stud. 42, 375-393 (1975)

Mitra, T., Roy, S.: Optimal exploitation of renewable resources under uncertainty and the extinction of species. Econ. Theory 28, 1-23 (2006)

Mitra, T., Sorger, G.: Extinction in common property resource models: an analytically tractable example. Econ. Theory 57, 41-57 (2014)

Nowak, A.: On perfect equilibria in stochastic models of growth with intergenerational altruism. Econ. Theory 28, 73-83 (2006)

Pardoux, E., Peng, S.: Adapted solution of a backward stochastic differential equation. Syst. Control Lett. 14, 55-61 (1990)

Peng, S.: A general stochastic maximum principle for optimal control problems. SIAM J. Control Optim. 28, 966-979 (1990)

Polyanin, A.D., Zaitsev, V.F.: Handbook of Exact Solutions for Ordinary Differential Equations. CRC Press, Boca Raton (1995)

Ramsey, F.P.: Mathematical theory of saving. Econ. J. 38, 543-559 (1928)

Reinganum, J.F., Stokey, N.L.: Oligopoly extraction of a common property natural resource: the importance of the period of commitment in dynamic games. Int. Econ. Rev. 26, 161-173 (1985)

Rincón-Zapatero, J.P.: Characterization of Markovian equilibria in a class of differential games. J. Econ. Dyn. Control 28, 1243-1266 (2004)

Rincón-Zapatero, J.P., Martínez, J., Martín-Herrán, G.: New method to characterize subgame perfect Nash equilibria in differential games. J. Optim. Theory Appl. 96, 377-395 (1998)

Shimomura, K.: The feedback equilibria of a differential game of capitalism. J. Econ. Dyn. Control 15, 317-338 (1991)

Sorger, G.: Markov-perfect Nash equilibria in a class of resource games. Econ. Theory 11, 79-100 (1998)

Sundaram, R.K.: Perfect equilibrium in non-randomized strategies in a class of symmetric dynamic games. J. Econ. Theory 47, 153-177 (1989)

Tsutsui, S., Mino, K.: Nonlinear strategies in dynamic duopolistic competition with sticky prices. J. Econ. Theory 52, 136-161 (1990) 
Van Long, N.: Dynamic games in the economics of natural resources: a survey. Dyn. Games Appl. 1, 115-148 (2011)

Yong, J., Zhou, X.Y.: Stochastic Controls. Hamiltonian Systems and HJB Equations. Springer, New York (1999)

Zhiyuan, H.: A comparison theorem for solutions of stochastic differential equations and its applications. Proc. Am. Math. Soc. 91, 611-617 (1984) 
Reproduced with permission of the copyright owner. Further reproduction prohibited without permission. 